\title{
FIXED POINT THEOREMS FOR VARIOUS CLASSES OF 1-SET-CONTRACTIVE AND 1-BALL-CONTRACTIVE MAPPINGS IN BANACH SPACES
}

BY

\author{
W. V. PETRYSHYN( $\left.{ }^{1}\right)$
}

\begin{abstract}
Let $X$ be a real Banach space, $D$ a bounded open subset of $X$, and $\bar{D}$ the closure of $D$. In $\S 1$ of this paper we establish a general fixed point theorem (see Theorem 1 below) for 1-set-contractions and 1-ball-contractions $T: \bar{D} \rightarrow X$ under very mild conditions on $T$. In addition to classical fixed point theorems of Schauder, Leray and Schauder, Rothe, Kransnoselsky, Altman, and others for $T$ compact, Theorem 1 includes as special cases the earlier theorem of Darbo as well as the more recent theorems of Sadovsky, Nussbaum, Petryshyn, and others (see $\S 1$ for further contributions and details) for $T h$-set-contractive with $k<1$, condensing, and 1 -set-contractive. In $\S \S 2,3,4$, and 5 of this paper Theorem 1 is used to deduce a number of known, as well as some new, fixed point theorems for various special classes of mappings (e.g. mappings of contractive type with compact or completely continuous perturbations, mappings of semicontractive type introduced by Browder, mappings of pseudo-contractive type, etc.) which have been recently extensively studied by a number of authors and, in particular, by Browder, Krasnoselsky, Kirk, and others (see $\$ 1$ for details),
\end{abstract}

Introduction. Let $X$ be a real Banach space, $D$ a bounded open subset of $X$, $\bar{D}$ and $\partial D$ the closure and the boundary of $D$, respectively. The object of this paper is two-fold.

First, in $\$ 1$ we extend our main fixed point result (see Theorem $7^{\prime}$ in Petryshyn [34]) by proving (see Theorem 1 below) that if $T: \bar{D} \rightarrow X$ is either a 1-set or 1 -ball contraction which satisfies the Leray-Schauder condition on $\partial D$, then $T$ has a fixed point in $\bar{D}$ if and only if $T$ satisfies condition (c). As will be seen, Theorem 1 unifies and extends in some cases to nonconvex domains and/or to more general boundary conditions a number of known, as well as some new, fixed

Received by the editors October 4, 1971 and, in revised form, November 2, 1972. AMS (MOS) subject classifications (1970)。 Primary 47H10; Secondary $47 \mathrm{H} 99$.

Key words and phrases. Fixed point theorems, topological degree, $k$-set-contractions, $k$-ball-contractions, set-condensing and ball-condensing mappings, mappings of contractive, semicontractive, and pseudo-contractive type.

(1) Supported in part by NSF Grant GP-20228 and in part by the Research Council of Rutgers University while the author was on the faculty research fellowship during the academic year 1970-1971. 
point theorems for special classes of mappings. In addition to fixed point theorems of [40], [38], [27], [1] and others ([20], [48]) for $T$ compact, Theorem 1 includes as special cases the earlier result of Darbo [9] for $k$-set-contractions with $k<1$ as well as the more recent theorems of Sadovsky [39], Furi and Vignoli [17] and Nussbaum [31] for $T$ ball-condensing and set-condensing and $D$ also convex. The result of Browder [7] for $T: \bar{D} \rightarrow \bar{D}$ a 1 -set-contraction with $D$ convex and $(I-T)(\bar{D})$ closed, and a theorem of Edmunds and Webb [11] for $X$ a Hilbert space and $T: X \rightarrow X$ either 1 -ball or 1 -set contractive with $(I-T)(\bar{B}(0, r))$ closed for each $r>0$ follow also from Theorem 1 .

Second, in $\$ \$ 2$ to 5 we apply Theorem 1 to the derivation of fixed point theorems (some new and some old) for various special classes of maps that have been recently studied by a number of authors and which, as will be seen, are either 1 -set or 1 -ball contractive. Our discussion in these sections underlines; in addition to generality, the unifying aspect of Theorem 1. We remark specifically that, even for these special cases, some of our results are more general than those obtained by other authors, usually by different methods.

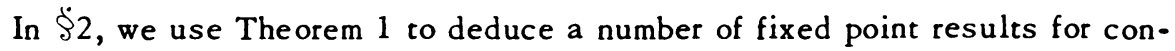
tractive, nonexpansive, and generalized contractive maps with compact and/or completely continuous perturbations. Some of the results in this section have been obtained earlier by other authors (see Remarks), and are in fact special cases of certain results deduced in $\$ 3$ for mappings of semicontractive type. We specifically include them in this section to indicate the history and the way the classical fixed point principles of Banach and Schauder have been extended recently to more general classes of mappings.

In $\$ 3$ we obtain a number of results for semicontractive maps introduced by Browder [4] and further studied by Browder, Kirk, Petryshyn, Webb, Nussbaum, and others (see Remarks). In $\$ 4$ we discuss further generalizations of Theorem 1 and apply these results to pseudo-contractive maps introduced by Browder and Petryshyn [8] in case of Hilbert spaces and by Browder [5] in case of Banach spaces. It will be seen in $\$ 4$ that when $X$ is a Hilbert space the definitions used in [8] and in [5], although seemingly different, are equivalent. In $\$ 5$ we use Theorem 1 to deduce fixed point theorems for maps satisfying suitable growth conditions. When $X$ is a Hilbert space our results include certain existence theorems for Hammerstein equations.

Finally we add that in our discussion of special cases of Theorem 1, we include a short survey of contributions by various authors which shows a step-bystep development of the fixed point theory for various special classes of mappings.

The outline of results presented in this paper has appeared in our note $A$ new fixed point theorem and its application, Bull. Amer. Math. Soc. 72 (1972), 225-229, 
where a degree theory for 1 -set and 1-ball contractive maps has been used to indicate the proof of Theorem 1 below.

1. Definitions and the main fixed point theorem. Let $X$ be a real Banach space and $D$ a bounded subset of $X$. Following [28] we define $\gamma(D)$, the set measure of noncompactness of $D$, to be inf $\{d>0 \mid D$ can be covered by a finite number of sets of diameter $\leq d\}$. It follows that $\gamma(D)=\gamma(\bar{D}), \gamma(\lambda D)=|\lambda| \gamma(D)$, $\gamma(D) \leq \gamma(Q)$ whenever $D \subset Q$ with $Q$ bounded, $\gamma(D \cup Q)=\max \{\gamma(D), \gamma(Q)\}$, and $\gamma(D)=0$ if and only if $\bar{D}$ is compact. Further, if $\overline{C O}(D)$ denotes the convex closure of $D$ and $D+Q=\{x+y \mid x \in D, y \in Q\}$, then $\gamma(D)=\gamma(\overline{c o}(D))$ and $\gamma(D+Q) \leq(D)+\gamma(Q)$ (see [9]).

Closely associated with $\gamma$ is the concept of a k-set-contraction $T: G \rightarrow X$ defined to be a bounded continuous map such that $\gamma(T(D)) \leq k \gamma(D)$ for each bounded $D \subset G$ and some constant $k \geq 0$. It follows that $C: \bar{G} \rightarrow X$ is compact if and only if $C$ is 0 -set-contractive, and that every Lipschitzian map $S: \bar{G} \rightarrow X$ with constant $l \geq 0$ is $l$-set-contractive. Clearly the map $T=S+C: \bar{G} \rightarrow X$ is also l-set-contractive. We shall also need the concept of a condensing map introduced first by Sadowsky [39] for ball-measure of noncompactness (see the definition below) and later by Furi and Vignoli [16] for the set-measure $\gamma$. A bounded continuous map $T$ of $G$ into $X$ is set-condensing (or densifying by [16]) if $\gamma(T(D))<\gamma(D)$ for each bounded $D \subset G$ with $\gamma(D) \neq 0$. It follows that every $k$ set-contractive map with $k<1$ is set-condensing and that every set-condensing map is 1-set-contractive but the reverse implications do not hold (see [31]).

In [39] Sadovsky used the ball-measure of noncompactness of $D$ defined by $\chi(D)=\inf \{r>0 \mid D$ can be covered by a finite number of balls with centers in $X$ and radius $r$. It is known ([17], [31]) that the measures $\gamma$ and $\chi$ are different although they have a good deal in common. We add that the above mentioned properties of $\gamma$ are also valid for $\chi$. As in the case of $\gamma$, corresponding to $\chi$ we have $k$-ball-contractions and ball-condensing maps. In general one cannot say much about the precise relationship between maps defined in terms of $\gamma$ and $\chi$. But, in case $T$ is a bounded linear map of $X$ to $X$, a number of results concerning this relationship has been recently obtained in [30], [36], [47]. The reason for introducing here $k$-ball-contractive and ball-condensing maps is that for the fixed point theory the same argument works for maps $T: \bar{D} \rightarrow X$ defined either in terms of $y$ or in terms of $\chi$. Furthermore, as we shall see, there are maps $T$ : $\bar{D} \rightarrow X$ which are set-condensing but it is unknown whether they are also ballcondensing and vice versa.

In what follows we shall need the following fixed point theorem which we state here as a lemma.

Lemma 1 (Petryshyn [34]). Let $D$ be a bounded open set in $X$ and let $T$ : 
$\bar{D} \rightarrow X$ be a 1 -set-contractive mapping for which the following bolds:

(a) There exists $x_{0} \in D$ such that if $T(x)-x_{0}=a\left(x-x_{0}\right)$ for some $x$ in $\partial D$, then $a \leq 1$.

(b) $(I-T)(\bar{D})$ is closed.

Then $T$ bas a fixed point in $\bar{D}$.

Remark 1.1. The arguments of [34] together with the degree theory for ball- condensing mappings in [40] show the validity of Lemma 1 for the case when $T$ : $\bar{D} \rightarrow X$ is 1 -ball-contractive.

Using Lemma 1 and Remark 1.1 we now give a simple proof of the following fixed point theorem for 1 -set and 1 -ball contractions which, as will be seen in $\$ \$ 2$ to 5 , unifies and extends most of the known fixed point theorems.

Theorem 1. Let $X$ be a real Banach space, $D$ a bounded open subset of $X$, and $T$ either a 1-set-contractive or a 1-ball-contractive map of $\bar{D}$ into $X$ for which condition (a) of Lemma 1 bolds. Then $T$ has a fixed point in $\bar{D}$ if and only if $T$ satisfies the following condition:

(c) If $\left\{x_{n}\right\}$ is any sequence in $\bar{D}$ such that $x_{n}-T\left(x_{n}\right) \rightarrow 0$ as $n \rightarrow \infty$, then there exists $x^{\prime} \in \bar{D}$ with $x^{\prime}-T\left(x^{\prime}\right)=0$.

Proof. It is clear that condition (c) holds if $T$ has a fixed point in $\bar{D}$.

We will prove the converse. For each $t_{n}$, with $0<t_{n}<1$ and $t_{n} \rightarrow 1$ as $n \rightarrow \infty$, consider the mapping $T_{n}: \bar{D} \rightarrow X$ defined by $T_{n}(x)=t_{n} T(x)+\left(1-t_{n}\right) x_{0}$. The properties of the measures $\gamma$ and $\chi$ imply that, for each fixed $n, T_{n}$ is either $t_{n}$-set-contraction or $t_{n}$-ball-contraction, depending on $T$. Hence, for each fixed $n,\left(I-T_{n}\right)(\bar{D})$ is a closed set since, as was shown in [29], $I-T_{n}$ is proper; moreover, if $T_{n}(x)-x_{0}=\lambda\left(x-x_{0}\right)$ for some $x$ in $\partial D$, then $T(x)-x_{0}=$ $\lambda t_{n}^{-1}\left(x-x_{0}\right)$ and therefore condition (a) implies that $\lambda t_{n}^{-1} \leq 1$, i.e., $\lambda \leq t_{n}<1$ for $n \geq 1$. Hence, by Lemma 1 and Remark 1.1, for each $n$ there exists an element $x_{n}$ in $D$ such that $T_{n}\left(x_{n}\right)=x_{n}$. Consequently, since $T$ and $\left\{x_{n}\right\}$ are bounded and $t_{n} \rightarrow 1$ as $n \rightarrow \infty, x_{n}-T\left(x_{n}\right)=\left(t_{n}-1\right) T\left(x_{n}\right)+\left(1+t_{n}\right) x_{0} \rightarrow 0$ as $n \rightarrow \infty$. This and condition (c) imply the existence an $x^{\prime}$ in $\bar{D}$ such that $x^{\prime}-$ $T\left(x^{\prime}\right)=0$. Q.E.D.

It is obvious that condition (b) of Lemma 1 implies condition (c) of Theorem 1 but an example will show in $\$ 2$ that if $D$ is also convex and $T$ is a generalized contraction of $\bar{D}$ into $\bar{D}$ in the sense of Belluce and Kirk [2], then T satisfies condition (c) but it is unknown whether condition (b) holds for this class of mappings. In case $T$ is a set-condensing or ball-condensing (and, in particular, $k$-set or $k$ ball contractions with $0 \leq k<1$ ), the sufficency part of Theorem 1 remains valid without the assumption of condition (c) for the latter is always true for this class of mappings. 
Note that condition (a) is implied by the assumption

$$
\|T(x)-x\|^{2} \geq\left\|T(x)-x_{0}\right\|^{2}-\left\|x-x_{0}\right\|^{2} \text { for } x \in \partial D
$$

used in [1] for the case when $D=B(0, r)$ and $x_{0}=0$ and in [20] for the case when $D=B\left(x_{0}, \tau\right)$. In case $0 \in D$, then

$$
(T(x), J(x)) \leq(x, J(x)) \text { for } x \in \partial D
$$

also implies (a), where $J$ is a duality map of $X \rightarrow 2^{X^{*}}$ corresponding to a given gauge function $\mu$, i.e., $J(x)=\left\{w \in X^{*} \mid(w, x)=\|w\|\|x\|,\|w\|=\mu(\|x\|)\right\}$, where $\mu: R^{+} \rightarrow R^{+}$is a strictly increasing function with $\mu(0)=0$ and $\mu(t) \rightarrow \infty$ as $t \rightarrow \infty$. If in Theorem $1, D$ is convex, then (a) is implied by

$$
T(\partial D) \subset \bar{D} \text { and, in particular, by } T(\bar{D}) \subset \bar{D} \text {. }
$$

The above remarks justify our assertion in the introduction that the classical fixed point theorems for $T$ compact, and the more recent theorems for $T$ setcondensing or ball-condensing, are special cases of Theorem 1.

2. Applications to contractive type maps with perturbations. In this section we indicate the generality of Theorem 1 and its unifying aspect by using it to deduce a number of fixed point theorems for operators of contractive type and the ir perturbations by compact and/or completely continuous maps. Some of the theorems mentioned in this section were obtained earlier by other authors, but usually by methods which are different from ours and sometimes under conditions which are more restrictive than those imposed here (see Remarks). Although a few fixed point theorems stated in this section are special cases of some results which will be deduced in $\$ 3$ for maps of semicontractive type, we specifically include them in this section to indicate the ir chronological development by various authors and the way the classical fixed point principles of Banach and Schauder have been extended step by step to more general classes of mappings.

Let $D$ be a subset of $X$ and let $S$ be a map of $\bar{D}$ into $X$ such that

$$
\|S(x)-S(y)\| \leq \alpha\|x-y\| \text { for } x, y \text { in } \bar{D} \text { and some } \alpha \geq 0 .
$$

Then $S$ is called contractive if $a<1$ and nonexpansive if $\alpha=1$. We shall also be concerned with a class of the so-called generalized contractive mappings $S$ : $\bar{D} \rightarrow X$ introduced by Belluce and Kirk [2] and defined to be such that to each $x$ in $\bar{D}$ there exists a number $a(x)<1$ with

$$
\|S(x)-S(y)\| \leq \alpha(x)\|x-y\| \text { for each } y \text { in } \bar{D} .
$$

It was noted in [2] that generalized contractions provide an example of a class of mappings of "diminishing orbital diameters" and thus that the fixed point theorem 
obtained for mappings of this latter type applies to generalized contractions. We show in this section that the fixed point theorem of Belluce and Kirk for generalized contraction is also deducible from Theorem 1. We add that the main motivation for the study of generalized contractions stems from the fact (see [25]) that if $D$ is a bounded open convex subset of $X$ and $T: D \rightarrow X$ is continuously $F$ differentiable on $D$, then $T$ is a generalized contraction on $D$ if and only if $\left\|T_{x}^{\prime}\right\|<1$ for each $x$ in $D$, where $T_{x}^{\prime}$ is the Fréchet derivative of $T$ at $x$ in $D$.

The first consequence of Theorem 1 is the following result which is new in the sense that, unlike other authors (see Remark 2.1), we do not require $D$ to be convex. Recall that $P: G \subset X \rightarrow X$ is compact if $P$ is continuous and $\overline{P(A)}$ is compact if $A \subset G$ is bounded.

Theorem 2.1. Let $D \subset X$ be bounded and open, $S: \bar{D} \rightarrow X$ contractive, and $C: \bar{D} \rightarrow X$ compact. If for some $x_{0} \in D$ the map $T=S+C$ satisfies the condition

$$
T(x)-x_{0} \neq \lambda\left(x-x_{0}\right) \quad \text { for } x \in \partial D \text { and } \lambda>1,
$$

then $T$ bas a fixed point in $D$.

Proof. It follows from our discussion in $\oint_{1}$ that $T=S+C: \bar{D} \rightarrow X$ is k-setcontractive with $k=\alpha<1$ and thus 1 -set-contractive. Furthermore, $T$ satisfies conditions (a) and (c) since (a) is equivalent to condition (LS) while (c) follows immediately from the structure of $T$. Hence, by Theorem $1, T$ has a fixed point.

Remark 2.1. Theorem 2.1 was established first in [26] under the additional hypothesis that $D$ is convex and that

$$
S(x)+C(y) \in \bar{D} \quad \text { for } x \text { and } y \text { in } \bar{D} .
$$

In case $X$ is a Hilbert space and $D$ is also convex, Theorem 2.1 has been constructively established in [32] for $X$ separable and $T$ satisfying (a) on $\partial D$, and independently in [50] for $X$ nonseparable and $T(\bar{D}) \subset \bar{D}$. In case $D$ is a ball $B(0, r)$, Theorem 2.1 has been established independently in [49] for a general Banach space with $T(\bar{D}) \subset \bar{D}$ and in [32] for a Banach $\pi_{1}$-space with a weakly continuous duality map. Further studies have been made in [14], [39], [7], [37], and others (see [15]). It should be added that since $T=S+C$ is $k$-set-contractive with $k<1$, Theorem 2.1 for the case when $D$ is convex and $T(\bar{D}) \subset \bar{D}$ is deducible from the theorem of Darbo [9] proved in 1955 which has unfortunately escaped the notice of researchers in this field until 1969 (see [29]):

Theorem 2.2. Let $D \subset X$ be bounded and open, $S: \bar{D} \rightarrow X$ nonexpansive, and $C: \bar{D} \rightarrow X$ compact. If $T=S+C$ satisfies conditions (a) and (c) of Theorem 1 , then $T$ bas a fixed point. 
Proof. Since $S: \bar{D} \rightarrow X$ is nonexpansive and $C$ is compact, $T=S+C$ : $\bar{D} \rightarrow X$ is 1 -set-contractive and hence Theorem 2.2 follows from Theorem 1 .

We say that a map $P: G \rightarrow X$ is demiclosed if $\left\{x_{n}\right\} \subset G$ is such that $x_{n} \rightarrow x$ $\in G$ and $P\left(x_{n}\right) \rightarrow f$ in $X$, then $P\left(x_{0}\right)=f$, where $\rightarrow$ and $\rightarrow$ denote the weak and the strong convegence in $X$, respectively. It was shown by Browder [6] that if $X$ is uniformly convex, $D$ convex and $T: \bar{D} \rightarrow X$ nonexpansive, then $P=I-T$ is demiclosed and in particular, condition (c) holds. Hence, an immediate consequence of Theorem 2.2 is the following corollary.

Corollary 2.1. If $X$ is uniformly convex, $D$ a bounded open convex subset of $X$, and $T: \bar{D} \rightarrow X$ a nonexpansive map which satisfies condition (a) on $\partial D$, then $T$ has a fixed point.

Remark 2.2. In case $T(D) \subset \bar{D}$, Corollary 2.1 has been first established independently in [3], [22], [19]. In its present form Corollary 2.1 has been obtained by Browder [6] as a special case of a more general theorem for maps of semicontractive type which we shall discuss in $\$ 3$. We add in passing that the arguments of Browder are not only different from ours but are also lengthy and quite complicated.

If in Theorem 2.2 we omit the requirement that $T$ satisfy condition (c), then $T$ may not have a fixed point in $\bar{D}$. In fact, it was shown in [4] that if $X=l_{2}$ and $D=B(0,1) \subset l_{2}$, then $S: \bar{B} \rightarrow l_{2}$ and $C: \bar{B} \rightarrow l_{2}$ given by

$$
S(x)=\left(0, x_{1}, x_{2}, x_{3}, \ldots\right), \quad C(x)=\left(1-\|x\|^{2}, 0,0,0, \ldots\right)
$$

is nonexpansive and compact, respectively; $T=S+C$ maps $\bar{B}$ into $\bar{B}$ (i.e., $T$ is 1 -set-contractive and satisfies condition (a)) but $T$ has no fixed points in $\bar{B}$. Consequently, some additional condition has to be imposed for $T=S+C$ to have a fixed point. Condition (c) is the weakest such condition.

However, the following theorem is valid for uniformly convex Banach spaces and completely continuous perturbations, where $C: G \rightarrow X$ is said to be completely continuous (or strongly continuous) if for any $\left\{x_{n}\right\} \subset G$ such that $x_{n}-x_{0}$ in $G$, $C\left(x_{n}\right) \rightarrow C\left(x_{0}\right)$ in $X$ as $n \rightarrow \infty$.

Theorem 2.3. Let $X$ be a uniformly convex Banach space, $D$ a bounded open convex subset of $X, S$ a nonexpansive map of $\bar{D}$ into $X$, and $C$ a completely continuous map of $\bar{D}$ into $X$. If the mapping $T=S+C: \bar{D} \rightarrow X$ satisfies condition (a) of Theorem 1 , then $T$ bas a fixed point in $\bar{D}$.

Proof. Since $X$ is uniformly convex (and thus reflexive) and $C$ is completely continuous on $\bar{D}, \gamma(C(A))=0$ for each subset $A$ of $\bar{D}$. Hence $T=S+C$ is a 1 set-contractive map of $\bar{D}$ into $X$. Furthermore, $T$ satisfies condition (c) on $\bar{D}$. 
Indeed, if $\left\{x_{n}\right\}$ is any sequence in $\bar{D}$ such that $x_{n}-T\left(x_{n}\right) \rightarrow 0$, then assuming without loss of generality that $x_{n}-x_{0}$ in $\bar{D}$ and using the complete continuity of $C$ we see that $C\left(x_{n}\right) \rightarrow C\left(x_{0}\right)$ as $n \rightarrow \infty$ and, therefore, $x_{n}-S x_{n}=x_{n}-$ $T\left(x_{n}\right)+C\left(x_{n}\right) \rightarrow C\left(x_{0}\right)$ as $n \rightarrow \infty$. Since $l-S$ is demiclosed, it follows that $x_{0}-S\left(x_{0}\right)=C\left(x_{0}\right)$, i.e., $x_{0}-T\left(x_{0}\right)=0$ and $T$ satisfies condition (c). Hence Theorem 2.3 follows from Theorem 1. Q.E.D.

Remark 2.3. In case $X$ is a reflexive Banach space with a single-valued weakly continuous duality mapping and $T$ is semicontractive (and thus includes maps of the form $S+C$; see $\$ 3$ ), Theorem 2.3 has been first established by Browder [4] but under the condition that $T$ is defined on all of $X$ and that $T$ satisfies condition $(K)$ on $\bar{D}$. Independently of [4], Edmunds [12] has proved Theorem 2.3 for Hilbert spaces under condition $(\mathrm{K})$. In case $D$ is a ball $B(0, r)$ in a reflexive $\pi_{1}$-space $X$ such that $X$ has a single-valued weakly continuous duality mapping and Property $(H)$ (i.e., if $x_{n}-x$ in $X$ and $\left\|x_{n}\right\| \rightarrow\|x\|$ as $n \rightarrow \infty$, then $x_{n} \rightarrow x$ in $X$ ), Theorem 2.3 has been established by Petryshyn [32]. In case $X$ is a Hilbert space and $T(\bar{D}) \subset \bar{D}$, Theorem 2.3 has also been proved in [50] by rather simple arguments (see also [37], [21], [10], [11]). The subsequent generalizations of the results of [4], [12], [32], [50] which include Theorem 2.3 in its present form have since been obtained by various authors. The more general latter results will be discussed in the succeeding sections.

Next we show that the condition on $X$ in Corollary 2.1 can be relaxed if we strengthen the condition on $T$.

Corollary 2.2. Let $X$ be a reflexive Banach space and $D$ a bounded open convex subset of $X$. If $T$ is a generalized contraction of $\bar{D}$ into $\bar{D}$, then $T$ bas a (unique) fixed point in $\bar{D}$.

Proof. Since $T$ is obviously 1 -set-contractive and the condition $T(\bar{D}) \subset \bar{D}$ implies the condition (a) of Theorem 1 for any $x_{0}$ in $D$, to prove Corollary 2.2 it suffices to show that $T$ satisfies condition (c) of Theorem 1. This we prove in the lemma below using a variant of the technique first employed by Kirk [23] under the assumption that $T$ is defined on all of $X$ (see also Fitzpatrick [13]).

Lemma 2.1. If $X$ is a reflexive Banach space, $D$ an open boundèd convex subset of $X$, and $T$ a generalized contraction of $\bar{D}$ into $\bar{D}$, then $T$ satisfies condition (c) on $\bar{D}$.

Proof. Let $\left\{x_{j}\right\} \subset \bar{D}$ be a sequence such that $x_{j}-T\left(x_{j}\right) \rightarrow 0$ as $j \rightarrow \infty$. Since $X$ is reflexive, $\bar{D}$ a bounded closed convex subset of $X$, and $\left\{x_{j}\right\} \subset \bar{D}$, we may assume that $x_{j} \rightarrow x_{0}$ in $\bar{D}$. We claim that $\left\{x_{j}\right\}$ converges strongly (necessarily to $\left.x_{0}\right)$ so that $x_{0}-T\left(x_{0}\right)=0$, i.e., $T$ satisfies condition (c). 
Let $R=\left\{r>0 \mid\right.$ there exists an integer $n>1$ such that $\bar{D} \cap\left\{\bigcap_{j \geq n} B\left(x_{j}, r\right)\right\}$ $\neq \varnothing\}$. Note that $R \neq \varnothing$ since $D$ is bounded and $\left\{x_{j}\right\}$ lies in $\bar{D}$. Thus, we may define $r_{0}=\inf \{r \mid r \in R\}$ and note that for each $\epsilon>0$, there exists a $k=k(\epsilon)$ such that

$$
C_{k}^{\epsilon}=\bar{D} \cap\left\{\bigcap_{j \geq k} B\left(x_{j}, r_{0}+\epsilon\right)\right\} \neq \varnothing .
$$

Since, for fixed $\epsilon,\left\{C_{k}^{\epsilon}\right\}$ is a monotonically increasing sequence of bounded, closed, convex, and nonempty subsets of $\bar{D}$ we see that

$$
C^{\epsilon}=\bigcup_{k=1}\left[\bar{D} \cap\left\{\bigcap_{j \geq k} B\left(x_{j}, x_{0}+\epsilon\right)\right\}\right]
$$

is a nonempty closed convex subset of $\bar{D}$ with the property that the family $F=\left\{C^{\epsilon} \mid \epsilon>0\right\}$ is a collection of nonempty weakly compact subsets of $\bar{D}$ having the finite intersection property. Hence, by the reflexivity of $X, \bigcap_{\epsilon>0} C^{\epsilon} \neq \varnothing$ and therefore there exists an element $\tilde{x} \in \bigcap_{\epsilon>0} C^{\epsilon} \subset \bar{D}$.

Let $r_{j}=\left\|x_{j}-T\left(x_{j}\right)\right\|$ for each $j$ and note that since $\alpha(\tilde{x})<1$ we can choose $\epsilon>0$ and $\delta>0$ such that $\beta=\alpha(\tilde{x})\left(r_{0}+\epsilon\right)+\delta<r_{0}$. Now, since $r_{j} \rightarrow 0$ as $j \rightarrow \infty$, we can choose $N_{0}$ so large that $r_{j}<\delta$ and $\tilde{x} \in B\left(x_{j}, r_{0}+\epsilon\right)$ for $j \geq N_{0}$. Therefore, for all $j \geq N_{0}$ we have

$$
\begin{aligned}
\left\|T(\tilde{x})-x_{j}\right\| & \leq\left\|T(\tilde{x})-T\left(x_{j}\right)\right\|+\left\|T\left(x_{j}\right)-x_{j}\right\| \\
& \leq a(\tilde{x})\left\|\tilde{x}-x_{j}\right\|+r_{j} \leq \alpha(\tilde{x})\left(r_{0}+\epsilon\right)+r_{j} \leq \beta .
\end{aligned}
$$

Hence, $T(\tilde{x}) \in \bigcap_{B}\left(x_{j}, \beta\right)$ and $T(\tilde{x})$ also lies in $\bar{D}$, by the assumption that $T(\bar{D}) \subset \bar{D}$. Thus $\bar{D} \cap\left\{\bigcap_{j \geq N_{0}} B\left(x_{j}, \beta\right)\right\} \neq \varnothing$ with $\beta<r_{0}$, in violation of the definition of $r_{0}$. Hence, $r_{0}=0$ and thus for each $\epsilon>0$ there exists $k(\epsilon)>1$ such that $\left\|x_{j}-x_{i}\right\|<2 \epsilon$ for $i, j \geq k(\epsilon)$, i.e., $\left\{x_{j}\right\}$ is a Cauchy sequence which necessarily converges (strongly) to $x_{0}$. This and the continuity of $T$ imply that $x_{0}-T\left(x_{0}\right)$ $=0$. This completes the proof of Lemma 2.1 and of Corollary 2.2. Q.E.D.

Remark 2.4. Corollary 2.2 has been first obtained in [2] as a consequence of a fixed point theorem for mappings of "diminishing orbital diameters".

Remark 2.5. Since it is unknown whether for $T$ satisfying the conditions of Corollary 2.2 the set $(I-T)(\bar{D})$ is closed, we see that Theorem 1 is indeed a generalization of Theorem $7^{\prime}$ in [34] even for 1 -set-contractions.

Remark 2.6. Since for any given $f$ in $X$ the map $T_{f}=T+f: \bar{D} \rightarrow X$ is also a generalized contraction, Lemma 2.1 implies that $T$, satisfies condition (c) provided $f$ is such that $T_{f}(x) \in \bar{D}$ for each $x$ in $\bar{D}$. Moreover, it follows from the proof of Lemma 2.1 that we have not only verified condition (c) for $T$, but in fact have also shown that if $T_{f}(\bar{D}) \subset \bar{D}$ and $\left\{x_{n_{j}}\right\}$ is any sequence in $\bar{D}$ such that 
$x_{n_{j}} \rightarrow x_{0}$ in $\bar{D}$ and $x_{n_{j}}-T\left(x_{n_{j}}\right) \rightarrow f$ as $j \rightarrow \infty$, then $x_{n_{j}} \rightarrow x_{0}$ as $j \rightarrow \infty$ and $x_{0}-T\left(x_{0}\right)=f$.

In view of Remark 2.6, the following results are also valid for certain perturbed generalized contractions.

Theorem 2.4. Let $X$ be a reflexive Banach space, $D$ a bounded open convex subset of $X, S$ a generalized contraction of $\bar{D}$ into $X$, and $C$ a completely continuous mapping of $\bar{D}$ into $X$. If $T$ satisfies the condition

$$
S(x)+C(y) \in \bar{D} \text { for } x, y \text { in } \bar{D},
$$

then $T$ bas a fixed point in $\bar{D}$.

Proof. Since $T=S+C$ is 1 -set-contractive on $\bar{D}$ and $T(\bar{D}) \subset \bar{D}$, by Theorem 1 for $x_{0}=0$, it suffices to show that $T$ satisfies condition (c) on $\bar{D}$. Let $\left\{x_{n}\right\}$ be any sequence in $\bar{D}$ such that $x_{n}-T\left(x_{n}\right) \rightarrow 0$ as $n \rightarrow \infty$. Assuming that $x_{n} \rightarrow x_{0}$ in $\bar{D}$ and using the complete continuity of $C$ we see that $C\left(x_{n}\right) \rightarrow$ $C\left(x_{0}\right)$ and $x_{n}-S\left(x_{n}\right)=x_{n}-T\left(x_{n}\right)+C\left(x_{n}\right) \rightarrow C\left(x_{0}\right)$ as $n \rightarrow \infty$. Since, by condition $(\mathrm{K}),\left(S(x)+C\left(x_{0}\right)\right) \in \bar{D}$ for each $x$ in $\bar{D}$, Remark 2.6 implies that $x_{0}-S\left(x_{0}\right)$ $=C\left(x_{0}\right)$ or $x_{0}-T\left(x_{0}\right)=0$, i.e., $T$ satisfies condition (c). Hence $T$ has a fixed point in $\bar{D}$. Q.E.D.

If in Theorem 2.4 we assume that $D=B(0, r)$, then condition $(\mathrm{K})$ could be somewhat relaxed (see [25] for the case when $C=0$ ).

Theorem 2.5. Let $X$ be a reflexive Banach space, $D=B(0, r)$, and let $S$ : $\bar{B} \rightarrow X$ and $C: \bar{B} \rightarrow X$ be as in Theorem 2.4. If $T=S+C$ satisfies the condition (K1)

$$
S(x)+C(y) \in \bar{B} \text { for } x \text { in } \partial B \text { and } y \text { in } \bar{B} \text {, }
$$

then $T$ bas a fixed point in $\bar{B}$.

Proof. Let $T^{\prime}(x)=S^{\prime}(x)+C^{\prime}(x)$ for $x$ in $\bar{B}$, where $S^{\prime}(x)=(x+S(x)) / 2$ and $C^{\prime}(x)=C(x) / 2$ for $x$ in $\bar{B}$. It is easy to show that $S^{\prime}: \bar{B} \rightarrow X$ is a generalized contraction, $C^{\prime}$ is completely continuous, and $T^{\prime}$ and $T$ have the same fixed points in $\bar{B}$. Furthermore, $T^{\prime}(\bar{B}) \subset \bar{B}$. To verify the last assertion, set $\bar{x}=r x /\|x\|$ for $x$ in $\bar{B}$ with $x \neq 0$. Since $S$ is a generalized contraction on $\bar{B}$ and (K1) holds, we have

$$
\begin{aligned}
\left\|S^{\prime}(x)+C^{\prime}(y)\right\| & \leq\|(x+S(x)) / 2-S(\bar{x}) / 2+S(\bar{x}) / 2+C(y) / 2\| \\
& \leq 1 / 2\|x\|+1 / 2\|S(x)-S(\bar{x})\|+1 / 2\|S(\bar{x})+C(y)\| \\
& \leq 1 / 2\|x\|+1 / 2\|x-\bar{x}\|+1 / 2 r=1 / 2\|x\|+1 / 2(r-\|x\|)\|x /\| x\|\|+1 / 2 r \\
& =r, \quad \text { for all } y \text { in } \bar{s} \text { and all } x \text { in } \bar{B} \text { with } x \neq 0 .
\end{aligned}
$$


On the other hand, let $x^{\prime}$ be any point in $\partial B$. Then, by (2.2) and (K1), for any $y$ in $\bar{B}$ we have

$$
\begin{aligned}
\left\|S^{\prime}(0)+C^{\prime}(y)\right\| & =1 / 2\|S(0)+C(y)\| \\
& \leq 1 / 2\left\|S(0)-S\left(x^{\prime}\right)\right\|+1 / 2\left\|S\left(x^{\prime}\right)+C(y)\right\| \leq 1 / 2 a(0) r+1 / 2 r<r .
\end{aligned}
$$

Hence, $S^{\prime}+C^{\prime}$ satisfies condition (K) and therefore, by Theorem $2.4, T$ has a fixed point in $\bar{B}$. Q.E.D.

Remark 2.7. In case $C=0$, then condition (K1) reduces to the assumption that $S(\partial B) \subset \bar{B}$. For the latter case Theorem 2.5 has been established in [25].

Let us continue this section by observing that if in Theorem 2.4 the condition on $S$ is somewhat strengthened, then the assertion of Theorem 2.4 is valid even for compact $C$ and without the stringent condition $(K)$. Thus we get the following interesting result.

Theorem 2.6. Let $X$ be a reflexive Banach space, $D$ a bounded open convex subset of $X, C$ a compact mapping of $\bar{D}$ into $X$, and $S$ a uniformly strictly contractive on $\bar{D}$ relative to $X$ (see [23]), i.e., the map $S: X \rightarrow X$ bas the property that for each $x$ in $X$ there exists a number $a(x)<1$ such that

$$
\|S(x)-S(y)\| \leq a(x)\|x-y\| \text { for each } y \text { in } \bar{D} \text {. }
$$

If $T=S+C: \bar{D} \rightarrow X$ satisfies condition (a) of Theorem 1 on $\partial D$, then $T$ bas a fixed point in $\bar{D}$.

Proof. Since $T=S+C$ is 1 -set-contractive and $T$ satisfies condition (a) of Theorem 1, to prove Theorem 2.6 it suffices to show that $T$ satisfies condition (c) on $\bar{D}$. Let $\left\{x_{n}\right\}$ be any sequence in $\bar{D}$ such that $x_{n}-T\left(x_{n}\right) \rightarrow 0$ as $n \rightarrow \infty$. Since $\left\{x_{n}\right\}$ is bounded and $C: \bar{D} \rightarrow X$ is compact we may assume that $C\left(x_{n}\right) \rightarrow f$ in $X$ for some $f$ in $X$. But then $x_{n}-S\left(x_{n}\right)=x_{n}-T\left(x_{n}\right)+C\left(x_{n}\right) \rightarrow f$ or $x_{n}-$ $F\left(x_{n}\right) \rightarrow 0$ as $n \rightarrow \infty$, where $F: X \rightarrow X$ is defined by $F(x)=S^{n}(x)+f$, for ${ }^{n}$ in $X$ with $F$ uniformly strictly contractive on $\bar{D}$ relative to $X$. Since $X$ is reflexive and $\bar{D}$ a bounded closed convex subset of $X$, there exists a subsequence $\left\{x_{n_{j}}\right\} \equiv$ $\left\{x_{j}\right\}$ for each $j$ and an element $x_{0}$ in $\bar{D}$ such that $x_{j} \rightarrow x_{0}$ and $x_{j}-F\left(x_{j}\right) \stackrel{n_{j}}{\rightarrow} 0$ as $j \rightarrow \infty$. It was shown by Kirk [23] that, under the above conditions on $F,\left\{x_{j}\right\}$ is necessarily a Cauchy sequence which converges strongly (necessarily to $x_{0}$ ) so that $x_{0}-F\left(x_{0}\right)=0$, i.e., $x_{0}-S\left(x_{0}\right)=f$. But then $C\left(x_{j}\right) \rightarrow C\left(x_{0}\right)=f$ as $j \rightarrow \infty$. Hence $x_{0}-T\left(x_{0}\right)=0$, i.e., $T$ satisfies condition (c) of Theorem 1 and so, by Theorem 2.2 or Theorem 1, T has a fixed point in $\bar{D}$. Q.E.D.

Remark 2.8. It is interesting to compare The ore $\mathrm{m} 2.6$ to Theorems 2.1 and 2.3 . 
3. Applications to mappings of semicontractive type. The object of this section is to deduce from Theorem 1 various fixed point theorems for mappings of semicontractive type introduced by Browder [6] and later also studied under various conditions by Kirk [23], Webb [45], [46], Browder [7], Petryshyn [32], Nussbaum [29], and others.

These mappings are the generalizations of the mappings studied in $\$ 2$ and they are obtained by intertwining mappings of contractive type and of compact or completely continuous type. It is in the first part of this section that the usefulness of the validity of Theorem 1 for 1 -ball-contractions will be apparent. We will deal with mappings for which it is easy to verify that they are 1-ball-contractions but for which it is unknown whether they are 1 -set-contractions.

To fix our terminology we consider the following definitions [4] (see also [45]).

Let $X$ be a Banach space, $D$ a bounded open subset of $X$, and $T$ a continuous map of $\bar{D}$ into $X$. Suppose there exists a continuous mapping $V$ of $X \times X$ into $X$ such that $T(x)=V(x, x)$ for $x$ in $\bar{D}$. Then

(1D) $T$ is strictly semicontractive if, for each fixed $x$ in $X, V(\cdot, x)$ is Lipschitzian with constant $k<1$ and $V(x, \cdot)$ is compact.

(2D) $T$ is weakly semicontractive if, for each $x$ in $X, V(., x)$ is nonexpansive and $V(x,$.$) is compact.$

(3D) $T$ is semicontractive if, for each fixed $x$ in $X, V(\cdot, x)$ is nonexpansive and $V(x,$.$) is completely continuous.$

Using the arguments analogous to those in [45] we now prove the following lemma.

Lemma 3.1. Let $X$ be a Banach space, $D$ a bounded open subset of $X$, and $T$ a continuous map of $\bar{D}$ into $X$ which is either strictly or weakly semicontractive. Then $T$ is $\lambda$-ball-contractive, where $\lambda=k$ or 1 depending on whether $T$ is strictly or weakly semicontractive.

Proof. Let $A$ be any subset of $\bar{D}$ and suppose that $\chi(A)=r>0$. Then given any $\epsilon>0$ we cover $A$ by a finite number of balls of radius $r+\epsilon$ with centers in $X$; say $B\left(x_{j}, r+\epsilon\right), 1 \leq j \leq k$, i.e., $A \subset \bigcup_{j=1}^{k} B\left(x_{j}, r+\epsilon\right)$. For each fixed $x$ in $X$, $V(x, A)$ is a precompact set in $X$ and so $\bigcup_{n=1}^{k} V\left(x_{n}, A\right)$ is also precompact. Therefore, for the given $\epsilon>0$, there exist points $z_{1}, \cdots, z_{p}$ in $X$ such that

$$
\bigcup_{n=1}^{k} V\left(x_{n}, A\right) \subset \bigcup_{j=1}^{p} B\left(z_{j}, \epsilon\right) .
$$

Now, given any $x$ in $A$ we choose $n$ such that $\left\|x-x_{n}\right\| \leq r+\epsilon$, and observe that

$$
\left\|V(x, x)-V\left(x_{n^{\prime}} x\right)\right\| \leq \lambda\left\|x_{n}-x\right\| \leq \lambda(r+\epsilon),
$$


where $\lambda$ equals either $k$ or 1 depending on $T$. Moreover, we may choose $j$ such that $\left\|V\left(x_{n}, x\right)-z_{j}\right\|<\epsilon$. Thus,

$$
\begin{aligned}
\left\|T(x)-z_{j}\right\| & =\left\|V(x, x)-z_{j}\right\| \\
& \leq\left\|V(x, x)-V\left(x_{n}, x\right)\right\|+\left\|V\left(x_{n}, x\right)-z_{j}\right\| \leq \lambda(r+\epsilon)+\epsilon \leq \lambda r+2 \epsilon .
\end{aligned}
$$

It follows from this that $T(A) \subset \bigcup_{j=1}^{p} B\left(z_{j}, \lambda r+2 \epsilon\right)$. In other words, $\chi(T(A)) \leq$ $\lambda r+2 \epsilon$ for any given $\epsilon>0$. Hence $\chi(T(A)) \leq \lambda \chi(A)$ with $\lambda=k$ if $T$ is strictly semicontractive and $\lambda=1$ if $T$ is weakly semicontractive. Q.E.D.

In view of Lemma 3.1, Theorem 1 implies the validity of the following new fixed point theorem under the general boundary condition (a) of Theorem 1 with $D$ not necessarily convex.

Theorem 3.1. Let $X$ be a Banach space, $D$ a bounded open subset of $X$, and $T$ a continuous map of $\bar{D}$ into $X$ such that condition (a) of Theorem 1 bolds. Suppose further that $T$ satisfies any one of the following two conditions:

(i) $T: \bar{D} \rightarrow X$ is strictly semicontractive.

(ii) $T: \bar{D} \rightarrow X$ is weakly semicontractive and $T$ satisfies condition (c) of Theorem 1 on $\bar{D}$.

Then, in either case, $T$ bas a fixed point in $\bar{D}$.

Proof. (i) Since $T: \bar{D} \rightarrow X$ is strictly semicontractive, Lemma 3.1 implies that $T$ is $k$-ball-contractive with $k<1$ and, in particular, 1-ball-contractive. Thus, in view of Theorem 1, it suffices to show that $T$ satisfies condition (c). Let $\left\{x_{n}\right\}$ be any sequence in $\bar{D}$ such that $g_{n} \equiv x_{n}-T\left(x_{n}\right) \rightarrow 0$ as $n \rightarrow \infty$. Since $x_{n}=g_{n}+T\left(x_{n}\right), \chi\left(\left\{g_{n}\right\}\right)=0$, and $T$ is $k$-ball-contractive with $k<1$, it follows that $\chi\left(\left\{x_{n}\right\}\right)=0$. Hence there exists a subsequence $\left\{x_{n_{j}}\right\}$ and an $x_{0}$ in $\bar{D}$ such that $x_{n_{j}} \rightarrow x_{0}$ as $j \rightarrow \infty$. This and the continuity of $T$ imply that $g_{n_{j}} \rightarrow x_{0}-T\left(x_{0}\right)=0$, i.e., condition (c) holds.

(ii) If $T$ satisfies condition (ii), then by Lemma 3.1 the map $T$ is 1 -ballcontractive and $T$ satisfies condition (c) by hypothesis. Hence Theorem 3.1 for $T$ satisfying either (i) or (ii) follows from Theorem 1 . Q.E.D.

If in (ii) we do not assume that $T$ satisfies condition (c), then for $T$ to have a fixed point in $\bar{D}$ we need to strengthen the conditions on $X, D$ and $V(., x)$. Indeed, using Theorem 1, Lemma 3.1, and the arguments of [4]. we have the following result.

Theorem 3.2. Let $X$ be a reflexive Banach space for which there exists a single-valued weakly continuous duality mapping $J$ of $X$ into $X^{*}$ with respect to some gauge function $\mu$. Let $\bar{D}$ be a bounded open convex subset of $X$ and $T: \bar{D} \rightarrow X$ a semicontractive mapping sucb that condition(a) of Theorem 1 bolds on $\partial D$. Then $T$ bas a fixed point in $\bar{D}$. 
Proof. Since $X$ is reflexive and, for each fixed $x$ in $X$, the map $V(x,$.$) is$ completely continuous, it follows that $V(x, \cdot)$ is also compact. Hence, by Lemma 3.1, T is 1-ball-contractive of $\bar{D}$ into $X$. To show that $T$ satisfies condition (c) of Theorem 1 , let $\left\{x_{n}\right\}$ be any sequence in $\bar{D}$ such that $x_{n}-T\left(x_{n}\right) \rightarrow 0$ as $n \rightarrow \infty$. We may assume that $x_{n}-x_{0}$ in $\bar{D}$. For all $x$ and $y$ in $\bar{D}$ let $V_{1}(x, y)$ $=x-V(x, y)$, where $V(x, x)=T(x)$ for $x$ in $\bar{D}$. Since $V$ is nonexpansive in the first variable, it follows that, for every $y$ in $X$,

$$
\begin{aligned}
& \left(V_{1}\left(y, x_{n}\right)-V_{1}\left(x_{n^{\prime}} x_{n}\right), J\left(y-x_{n}\right)\right) \\
& \quad=\left(y-x_{n^{\prime}} J\left(y-x_{n}\right)\right)-\left(V\left(y, x_{n}\right)-V\left(x_{n^{\prime}} x_{n}\right), J\left(y-x_{n}\right)\right) \geq 0 .
\end{aligned}
$$

Since $V_{1}\left(x_{n}, x_{n}\right)=x_{n}-T\left(x_{n}\right) \rightarrow 0, V_{1}\left(y, x_{n}\right) \rightarrow V_{1}\left(y, x_{0}\right)$ by the complete continuity of $V(y, \cdot)$, and $J\left(y-x_{n}\right) \rightarrow J\left(y-x_{0}\right)$ by the weak continuity of $J$, the passage to the limit in the above inequality implies that

$$
\left(V_{1}\left(y, x_{0}\right), J\left(y-x_{0}\right)\right)=\left(y-V\left(y, x_{0}\right), J\left(y-x_{0}\right)\right) \geq 0 \text { for all } y \text { in } X \text {. }
$$

We now let $z$ be an arbitrary element of $X$ and for each $t>0$ we set $y_{t}=x_{0}+$ tz. As $t \rightarrow 0, y_{t} \rightarrow x_{0}$ and therefore from the inequality $\left(y_{t}-V\left(y_{t}, x_{0}\right), J(t z)\right)$ $\geq 0$ and the fact that $J(t z)=\xi_{z}(t) J(z)$ with $\xi_{z}(t)>0$ it follows that $\left(y_{t}-\right.$ $\left.V\left(y_{t}, x_{0}\right), J(z)\right) \geq 0$. Passing to the limit in the last inequality as $t \rightarrow 0$ we get the relation

$$
\left(x_{0}-V\left(x_{0}, x_{0}\right), J(z)\right) \geq 0 \text { for all } z \text { in } X .
$$

Since, by the results in [7], $R(J)=X^{*}$, it follows that $T\left(x_{0}\right)=x_{0}$, i.e., $T$ satisfies condition (c). Q.E.D.

Remarks 3.1. If in Theorem 3.1 we assume that $D$ is also convex, then condition (a) is implied by the assumption that $T(\partial D) \subset \bar{D}$ and, in particular, by the condition that $T(\bar{D}) \subset \bar{D}$. Thus, Theorem 3.1 for $T$ satisfying condition (i) contains Theorem 2 of Webb [45] who generalized the results of [4] for the case when $D$ is also convex and $T(\bar{D}) \subset \bar{D}$.

3.2. Theorem 3.1 for $T$ satisfying condition (ii) contains Theorem 2 of Browder [4] which requires the additional hypotheses that $X$ be a reflexive Banach space with a weakly continuous duality mapping, that $T(\bar{D}) \subset \bar{D}$, and that $(I-T)(\bar{D})$ be closed.

3.3. Theorem 3.2, for the case when $T(\partial D) \subset \bar{D}$, contains Theorem 1 of Browder [4] which requires the additional hypothes is that $V \operatorname{map} X \times X$ into $\bar{D}$. Theorem 3.2 contains also Theorem 3 of Webb [45] established by him for the case when $T(\bar{D}) \subset \bar{D}$. Theorem 4 in [45] follows also from our Theorems 3.1 or 3.2.

Note that, since the semicontractive and weakly semicontractive mappings studied by the author in [32] were assumed to be defined only on a given ball 
$\bar{B}(0, r)$ ' in $X$ (and not on all of $X$ as in [4]), Theorems 6 and 7 in [32] cannot be deduced from Theorems 3.1 or 3.2 above for, in view of Lemma 3.1, the latter theorems were obtained under the essential assumptions that the given mappings were defined on all of $X$.

We now show that if we strengthen the compactness assumptions in the second variable of $V$, then the fixed point results analogous to Theorems 3.1 and 3.2 are also valid without the assumption that $T$ is defined on all of $X$, that $X$ has a weakly continuous duality mapping, or that $T(x)=V(x, x)$ satisfies condition (c) on $\bar{D}$. From our results we deduce as special cases the fixed point the orems of Browder [6] and Kirk [23].

We start with the following definitions due to Browder [6]. Let $D$ be a bounded open subset of a Banach space $X$ and $T$ a continuous map of $\bar{D}$ into $X$. Suppose there exists a continuous map $V: \bar{D} \times \bar{D} \rightarrow X$ such that $T(x)=V(x, x)$ for $x \in \bar{D}$. Then

(4D) $T$ is of strictly semicontractive type if, for each $x$ in $\bar{D}, V(\cdot, x)$ is Lipschitzian for some constant $k<1$ independent of $x$, and the map $x \rightarrow V(., x)$ is compact from $\bar{D}$ into the space of continuous mappings of $\bar{D}$ into $X$ with the uniform metric.

(5D) $T$ is of weakly semicontractive type if, for each $x$ in $\bar{D}, V(\cdot, x)$ is a nonexpansive map of $\bar{D}$ into $X$ and the map $x \rightarrow V(., x)$ of $\bar{D}$ into the space of continuous mappings of $\bar{D}$ into $X$ is compact.

(6D) $T$ is of semicontractive type if, for each $x$ in $\bar{D}, V(\cdot, x)$ is a nonexpansive map of $\bar{D}$ into $X$ and $V(x,$.$) is completely continuous from \bar{D}$ to $X$, uniformly for $x$ in $\bar{D}$.

We start with the following lemma whose simple proof is given in [29] and which we include here for the sake of completeness.

Lemma 3.2. Let $D$ be a bounded open subset of a Banach space $X$ and $T$ : $\bar{D} \rightarrow X$ a continuous mapping which is either of strictly or of weakly semicontrac. tive type. Then $T$ is $\lambda$-set-contractive with $\lambda=k$ or $\lambda=1$ depending on whether $T$ is of strictly or of weakly semicontractive type.

Proof. Let $A$ be any subset of $\bar{D}$. Since the map $x \rightarrow V(\cdot, x)$ is compact from $\bar{D}$ to $C(\bar{D}, X)$, the space of continuous mappings of $\bar{D}$ into $X$ with the uniform metric, it follows that the set $V(., A) \subset C(\bar{D}, X)$ is compact, where $V(., A)=$ $\{V(., x) \in C(\bar{D}, X) \mid x \in A\}$. Hence $V(., A)$ is totally bounded in $C(\bar{D}, X)$, i.e., to each $\epsilon>0$ there corresponds a finite set of points $x_{1}, \cdots, x_{n}$ in $A$ such that for every $x$ in $A$ there exists some $x_{j}, 1 \leq j \leq n$, such that

$$
\left\|V(\cdot, x)-V\left(\cdot, x_{j}\right)\right\|=\sup _{v \in \bar{D}}\left\|V(y, x)-V\left(y, x_{j}\right)\right\|<\epsilon .
$$


Hence $\sup _{y \in A}\left\|V(y, x)-V\left(y, x_{j}\right)\right\|<\epsilon$. Since $V(A, x)=V\left(A, x_{j}\right)+V(A, x)-$ $V\left(A, x_{j}\right)$, it follows that

$$
T(A)=V(A, A) \subset \bigcup_{j=1}^{n} N_{\epsilon}\left(V\left(A, x_{j}\right)\right)
$$

and therefore $\gamma(T(A)) \leq \max _{1 \leq j \leq n}\left\{\gamma\left(V\left(A, x_{j}\right)\right)+2 \epsilon\right\}$. But since $V\left(\cdot, x_{j}\right)$ is a $\lambda$ set-contraction with $\lambda=k$ or $\lambda=1, \gamma\left(V\left(A, x_{j}\right)\right) \leq \lambda y(A)$. Consequently, $\gamma(T(A)) \leq \lambda y(A)+2 \epsilon$ for each given $\epsilon>0$. Hence $T$ is $\lambda$-set-contractive on $\bar{D}$ with $\lambda=k$ or $\lambda=1$ depending on T. Q.E.D.

In view of Lemma 3.2, Theorem 1 implies the validity of the following new fixed point theorem.

Theorem 3.3. Let $D$ be a bounded open subset of the Banacb space $X$ and $T$ a continuous mapping of $\bar{D}$ into $X$ such that condition (a) of Theorem 1 bolds. Suppose further that $T$ satisfies any one of the following two conditions:

(i) $T$ is of strictly semicontractive type.

(ii) $T$ is of weakly semicontractive type and $T$ satisfies also condition (c) of Theorem 1 .

Then, in either case, $T$ bas a fixed point in $\bar{D}$.

Remark 3.4. Theorem 3.3 includes Theorem 5 of Browder [6] which requires the additional hypotheses that $D$ be also convex, $0 \in D$, and that $(I-T)(\bar{D})$ be also closed when $T$ is assumed to be only of weakly semicontractive type. We add in passing that Browder's method is different from the method used here. Theorem 3.3, although not explicitly stated, has been obtained in Petryshyn (see Theorem $7^{\prime}$ in [34]) under the stronger assumption that $(I-T)(\bar{D})$ is also closed in case $T$ is of weakly semicontractive type.

Now, if we assume that $X$ is also reflexive and $D$ is also convex, then every mapping $T: \bar{D} \rightarrow X$ of semicontractive type is also of weakly semicontractive type. To see the latter, it suffices to show that the map $x \rightarrow V(\cdot, x)$ of $\bar{D}$ into $C(\bar{D}, X)$ is compact, i.e., every sequence of elements in $V(., A)$ has a subsequence which converges to an element in $C(\bar{D}, X)$. Let $\left\{V\left(., y_{j}\right)\right\}$ be any sequence in $V(\cdot, A)$. Since $\left\{y_{j}\right\} \subset A$ and $A$ is a bounded set in a convex subset $D$ of a reflexive Banach space $X$, there exists a subsequence, which we again denote by $\left\{y_{j}\right\}$, and an element $y_{0}$ in $\bar{D}$ such that $y_{j} \rightarrow y_{0}$ as $j \rightarrow \infty$. Hence, since $T: \bar{D} \rightarrow X$ is of semicontractive type, to each $\epsilon>0$ there exists an $N>1$ such that

$$
\left\|V\left(x, y_{j}\right)-V\left(x, y_{0}\right)\right\|<\epsilon \text { for all } x \text { in } \bar{D} \text { and } j \geq N \text {. }
$$

This shows that $V\left(\cdot, y_{j}\right)$ converges to a mapping $V\left(\cdot, y_{0}\right)$ in $C(\bar{D}, X)$. Consequently, $T$ is of weakly semicontractive type. 
Now, it has been shown by Browder [6] that if we additionally assume that $X$ is uniformly convex, then $(I-T)(\bar{D})$ is a closed set in $X$. Since the latter fact implies condition (c), the preceding discussion shows that the following fixed point theorem of Browder (see Theorem 1 in [6]) follows as a special case of our Theorem 1.

Corollary 3.1. Let $X$ be a uniformly convex Banach space, $D$ a bounded open convex subset of $X$, and $T: \bar{D} \rightarrow X$ a map of semicontractive type which satisfies condition (a) of Theorem 1 on $\partial D$. Then $T$ bas a fixed point in $\bar{D}$.

In [23] Kirk extended the results of Browder [6] for semicontractive mappings to mappings of strongly semicontractive type. This extension, from the class of uniformly convex spaces to a much wider class of reflexive Banach spaces, requires the stronger version of semicontractiveness defined as follows:

(7D) A continuous mapping $T$ of $\bar{D}$ into $X$ is said to be of strongly semicontractive type relative to $X$ if there exists a mapping $V: X \times \bar{D} \rightarrow X$ with $T(x)=V(x, x)$ for $x$ in $\bar{D}$ such that for each $x$ in $\bar{D}$ the mapping $V(., x)$ is uniformly strictly contractive on $\bar{D}$ relative to $X$ (i.e., for each $x$ in $X$ there exists $0 \leq \alpha(x)<1$ such that $\|V(x, y)-V(z, y)\| \leq \alpha(x)\|x-z\|$ for each $z$ and $y$ in $\bar{D})$ and $V(x, \cdot)$ is completely continuous from $\bar{D}$ to $X$, uniformly for $x$ in $\bar{D}$.

This class of mappings generalizes mappings of the form $S+C$ with $S$ uniformly strictly contractive on $\bar{D}$ relative to $X$ and $C$ completely continuous on $\bar{D}$. As another consequence of Theorem 1 we deduce the following fixed point theorem obtained by Kirk [23] (for the case when $0 \in D$ ).

Corollary 3.2. Let $X$ be a reflexive Banach space, $D$ a bounded open convex subset of $X$, and $T: \bar{D} \rightarrow X$ of strongly semicontractive type relative to $X$ such that condition (a) of Theorem 1 bolds on $\partial D$. Then $T$ bas a fixed point in $\bar{D}$.

Proof. Since every $T: \bar{D} \rightarrow X$ of strongly semicontractive type relative to $X$ is necessarily of semicontractive type and since $X$ is reflexive and $D$ is also convex, the discussion preceding the statement of Corollary 3.1 and Lemma 3.2 show that $T$ is 1 -set-contractive. Moreover, the fact that $T$ satisfies condition (c) of Theorem 1 on $\bar{D}$ follows from Theorem 2 in [23]. Hence Corollary 3.2 follows from Theorem 1. Q.E.D.

Remark 3.5. We observe in passing that Corollary 3.2 is not an extension of Theorem 2.6.

We also note that if in definition (7D) we assume that $V: \bar{D} \times \bar{D} \rightarrow \bar{D}$ and that $T(x)=V(x, x)$ for $\propto x$ in $\bar{D}$, then the same arguments as those used in the proof of Lemma 2.1 show that $T$ satisfies condition (c) on $\bar{D}$. Hence in this case the assertion of Corollary 3.2 remains valid for $T: \bar{D} \rightarrow \bar{D}$ of a strongly 
semicontractive type (relative to $\bar{D}$ ) (i.e., without the assumption that for $y$ in $\bar{D}$ the map $V(., y)$ is defined on all $X)$.

We complete this section with the following discussion. In [29] Nussbaum generalized the fixed point theorem of Browder-Kirk-Göhde for nonexpansive mappings as well as a fixed point theorem of Browder [6] for maps of semicontractive type to locally almost nonexpansive mappings (lane mappings), where the latter is defined to be a continuous mapping $T$ of $\bar{D}$ into $X$ such that, given any $x$ in $\bar{D}$ and $\epsilon>0$, there exists a weak neighborhood $N_{x}$ of $x$ in $\bar{D}$ (depending also on $\epsilon$ ) for which

$$
\|T(x)-T(y)\| \leq\|x-y\|+\epsilon \text { for all } x, y \text { in } \mathrm{N}_{x} .
$$

It was shown in [29] that if $X$ is a reflexive Banach space, $D$ a bounded open convex subset of $X$, and $T$ a lane mapping of $\bar{D}$ into $X$, then $T$ is 1 -set-contractive; moreover, if $X$ is also uniformly convex, then $I-T$ is a demiclosed mapping of $\bar{D}$ into $X$.

In view of the above mentioned results, Theorem 1 implies the validity of the following generalization of a fixed point theorem for lane mappings obtained in [29].

Theorem 3.4. Let $X$ be a uniformly convex Banach space, $D$ a bounded open convex subset of $X, L$ a lane mapping of $\bar{D}$ into $X$, and $C$ a completely continuous mapping of $\bar{D}$ into $X$. If the map $T=L+C: \bar{D} \rightarrow X$ satisfies (a) of Theorem 1 on $\partial D$, then $T$ bas a fixed point in $\bar{D}$.

Proof. Note first that since $X$ is reflexive, $C: \bar{D} \rightarrow X$ is completely continuous, and $L: \bar{D} \rightarrow X$ is a lane mapping, $T=L+C$ is 1 -set-contractive. Furthermore, $T$ satisfies condition (c) on $\bar{D}$. Indeed, let $\left\{x_{n}\right\} \subset \bar{D}$ be such that $x_{n}-T\left(x_{n}\right) \rightarrow 0$. Since $D$ is a bounded convex subset of a reflexive Banach space $X$ and $C$ is completely continuous, we may assume that $x_{n} \rightarrow x_{0}$ in $\bar{D}$ and $C\left(x_{n}\right) \rightarrow C\left(x_{0}\right)$ in $X$. Hence $x_{n}-L\left(x_{n}\right)=x_{n}-T\left(x_{n}\right)+C\left(x_{n}\right) \rightarrow C\left(x_{0}\right)$ whence, since $x_{n} \rightarrow x_{0}$ and $I-L$ is demiclosed whenever $X$ is uniformly convex [29], we get $x_{0}-L\left(x_{0}\right)=C\left(x_{0}\right)$ or $x_{0}-T\left(x_{0}\right)=0$, i.e., $T$ satisfies (c) on $\bar{D}$. Hence, Theorem 3.4 follows from Theorem 1 .

Remark 3.6. For $C=0$ and $T(\partial D) \subset \bar{D}$, Theorem 3.4 has been proved in [29].

Remark 3.7. It was observed by the referee that one can show that $T=L$ $+C$ is also a lane map and so Theorem 3.4 can be deduced from the results on lane maps in [29] at least when $T(\partial D) \subset \bar{D}$. Although this observation is correct, the proof that $L+C$ is a lane map is not as easy as the referee seems to imply. On the other hand, our verification of condition (c) for perturbed map $T=L+C$ is straightforward. 
4. Further generalizations with applications to pseudo-contractive mappings. In $\$ 1$ we are concerned with the existence of fixed points for 1 -set-contractive or 1-ball-contractive mappings $T$ of $\bar{D}$ into $X$, that is, with the solvability of the equation

$$
\dddot{T}(x)-x=0 \quad(x \in \bar{D}) .
$$

In this section we discuss the solvability of the equation

$$
F(x)-A(x)=0 \quad(x \in \bar{D}),
$$

where $F$ and $A$ are suitable continuous mappings from a subset $\bar{D}$ of a real Banach space $X$ to another real Banach space $Y$. To obtain the solvability of equation (4.2), we first establish a slight generalization of Theorem 1 and then apply it to the solvability of equations involving various types of pseudocontractive mappings introduced by Browder and Petryshyn [8] for Hilbert spaces and later by Browder [5] for Banach spaces and further studied by Browder [7], Kirk [24], Petryshyn [36], Gatica and Kirk [18], and others.

Theorem 1'. Let $X$ and $Y$ be real Banach spaces, $G$ a bounded open sub. set of $X$, and $A$ a one-to-one continuous mapping of $\bar{G}$ into $Y$ such that $A(G)$ is bounded and open, $A(\bar{G})$ is closed in $Y$, and $A^{-1}$ is a continuous mapping of $A(\bar{G})$ into $X$. Let $F$ be a continuous mapping of $\bar{G}$ into $Y$ such that the mapping $F A^{-1}$ of $A(\bar{G}) \subset Y$ into $Y$ is either 1-set-contractive or 1.ball-contractive on $A(\bar{G})$. Suppose further that the following two conditions bold:

(a') There exists $y_{0}$ in $G$ such that if $F(y)-A\left(y_{0}\right)=a\left(A(y)-A\left(y_{0}\right)\right)$ bolds for some $y$ in $\partial G$, then $\alpha \leq 1$.

(c') If $\left\{y_{n}\right\}$ is any sequence in $\bar{G}$ such that $F\left(y_{n}\right)-A\left(y_{n}\right) \rightarrow 0$ as $n \rightarrow \infty$, then there exists $y^{\prime}$ in $\bar{G}$ such that $F\left(y^{\prime}\right)-A\left(y^{\prime}\right)=0$.

Then, in either case, the equation (4.2) bas a solution in $\bar{G}$.

Proof. If we set $D=A(G)$, then it follows from our conditions on $A$ and $F$ that $A(\bar{G})$ is a closed set in $Y, \bar{D}=A(\bar{G})$, and the mapping $T=F A^{-1}: \bar{D} \rightarrow Y$ satisfies all the conditions of Theorem 1. Indeed, since $F A^{-1}$ is either 1 -setcontractive or 1-ball-contractive, it suffices to verify that $T=F A^{-1}$ satisfies conditions (a) and (c) of Theorem 1.

Since $y_{0}$ lies in $G$, the point $x_{0}=A\left(y_{0}\right)$ lies in the open set $D$ and ( $\left.\mathrm{a}^{\prime}\right)$ implies that $T$ satisfies condition (a) on $\partial D$. In fact, if $T(x)-x_{0}=\alpha\left(x-x_{0}\right)$ for some $x$ in $\partial D$, then, by our conditions on $A, x=A(y)$ for some $y$ in $\partial G$ and consequently we have the relation $F(y)-A\left(y_{0}\right)=\alpha\left(A(y)-A\left(y_{0}\right)\right)$ for some $y$ in $\partial G$. Hence, condition ( $\mathrm{a}^{\prime}$ ) implies that $\alpha \leq 1$, i.e., $T$ satisfies (a) of Theorem 1 .

Suppose now that $\left\{x_{n}\right\}$ is a sequence in $\bar{D}$ such that $x_{n}-T\left(x_{n}\right) \rightarrow 0$ in $Y$ as $n \rightarrow \infty$. Since for each $n$ there corresponds a unique $y_{n}$ in $\frac{n}{G}$ such that 
$x_{n}=A\left(y_{n}\right)$ or $y_{n}=A^{-1}\left(x_{n}\right)$, it follows that $x_{n}-T\left(x_{n}\right)=A\left(y_{n}\right)-F\left(y_{n}\right) \rightarrow 0$ or $F\left(y_{n}\right)-A\left(y_{n}\right) \rightarrow 0$ as $n \rightarrow \infty$. Hence, condition (c') implies the existence of a point $y^{\prime}$ in $\bar{G}$ such that $F\left(y^{\prime}\right)-A\left(y^{\prime}\right)=0$ or $x^{\prime}-T\left(x^{\prime}\right)=0$ with $x^{\prime}=A\left(y^{\prime}\right) \in \bar{D}$, i.e., condition (c) holds.

Since $T=F A^{-1}: \bar{D} \rightarrow Y$ satisfies all the conditions of Theorem 1 , there exists a point $\tilde{x}$ in $\bar{D}$ such that $T(\tilde{x})=\tilde{x}$ or $F(\tilde{y})-A(\tilde{y})=0$ with $\tilde{y}=$ $A^{-1}(\tilde{x}) \in \bar{G}$. Q.E.D.

Remark 4.1. In the case when $Y=X$ and $A=I$, Theorem $1^{\prime}$ reduces to Theorem 1 for $T=F$.

Remark 4.2. The hypothes is that $F A^{-1}: \bar{D}=A(\bar{G}) \rightarrow Y$ is either 1 -set-contractive or 1-ball-contractive holds, in particular, when $F$ and $A$ are assumed to be such that any one of the following two conditions holds:

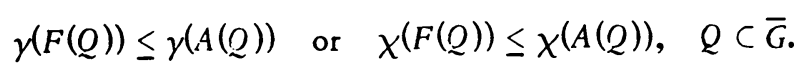

To see this suppose that $V$ is any subset in $\bar{D}$. Since $\bar{D}=A(\bar{G}), Q=A^{-1}(V)$ is a subset of $\bar{G}$. Hence, by the first inequality in (4.3), we have

$$
\gamma\left(F A^{-1}(V)\right)=\gamma\left(F\left(A^{-1}(V)\right)\right) \leq \gamma\left(A\left(A^{-1}(V)\right)\right)=\gamma(V),
$$

i.e., $F A^{-1}$ is 1 -set-contractive. A similar argument also shows that $F A^{-1}$ is 1 ball-contractive if the second inequality holds in (4.3).

Remark 4.3. If $G$ is also convex and the mapping $A: \bar{G} \rightarrow Y$ is such that $A(\lambda x+(1-\lambda) y)=\lambda A(x)+(1-\lambda) A(y)$ for all $x$ and $y$ in $\bar{G}$ and any $\lambda$ in $[0,1]$, then condition $\left(\mathrm{a}^{\prime}\right)$ of Theorem $1^{\prime}$ is implied by the requirement that $F(\partial G) \subset$ $A(\bar{G})$. Indeed, let $y_{0}$ be any poin in $G$ and suppose that $F(y)-A\left(y_{0}\right)=$ $\alpha\left(A(y)-A\left(y_{0}\right)\right)$ for some $y$ in $\partial G$. Then by our assumption, $F(y)=\alpha A(y)+(1-\alpha) A\left(y_{0}\right)$ lies in $\bar{D}$. Since $D$ is an open convex set, $A\left(y_{0}\right) \in D, A(y) \in \partial D$, and a may be assumed to be nonnegative, the fact that $\alpha A(y)+(1-\alpha) A\left(y_{0}\right) \in \bar{D}$ implies that $a \leq 1$.

In what follows we shall deduce from Theorems 1 and $1^{\prime}$ a number of fixed point theorems for the class of pseudo-contractive mappings which for the case of Banach spaces have been defined in [5] to be mappings $U: G \subset X \rightarrow X$ such that

$$
\|x-y\| \leq\|(1+r)(x-y)-r(U(x)-U(y))\| \text { for all } x, y \in G \text { and all } r>0 \text {. }
$$

This class of maps is more general than the class of nonexpansive maps and, as has been observed in [5], it has the useful property that $U$ is pseudo-contractive if and only if $I-U$ is accretive, i.e., $((I-U)(x)-(I-U)(y), J(x-y)) \geq 0$ for all $x, y \in G$, where $J$ is a normalized duality map of $X$ into $X^{*}$ (i.e., $J$ corresponds to the gauge function $\mu(t)=t$ ).

We add that when $X$ is a Hilbert space the notion of a pseudo-contractive 
mapping has been first introduced by Browder and Petryshyn [8] by defining it to be a mapping $U: G \rightarrow X$ such that

$$
\|U(x)-U(y)\|^{2} \leq\|x-y\|^{2}+\|(I-U)(x)-(I-U)(y)\|^{2} \text { for all } x, y \in G .
$$

It was shown in [8] that $U$ is pseudo-contractive (i.e., (4.5) holds) if and only if $I-U$ is monotone on $G$ (i.e., $I-U$ is accretive with $J=I$ ). Since $I-U$ is also monotone if and only if (4.4) holds we see that in the case of Hilbert spaces the definitions (4.4) and (4.5) are equivalent.

We note in passing that if we set $\lambda=r /(1+r)$, then (4.4) is equivalent to the requirement that

$$
(1-\lambda)\|x-y\| \leq\|(I-\lambda U)(x)-(I-\lambda U)(y)\| \text { for } x, y \in G \text { and } \lambda \in(0,1) \text {. }
$$

Now, the first consequence of Theorem $1^{\prime}$ is the following generalization of Theorem 1 in [18].

Theorem 4.1. Let $X$ be a Banach space, $G$ a bounded open subset of $X$ with 0 in $D$, and let $U$ be a map of $\bar{G}$ into $X$ such that

(i) $U$ is $k$-set-contractive on $\bar{G}$ for some $k \geq 0$.

(ii) $U$ is pseudo-contractive on $\bar{G}$.

(iii) $U$ satisfies condition (a) of Theorem 1 for $x_{0}=0$.

(iv) $U$ satisfies condition (c) of The orem 1 on $\bar{G}$. Then $U$ has a fixed point in $\bar{G}$.

Proof. To see that Theorem 4.1 follows from Theorem 1', define the mappings $F=\left(1-\lambda_{0}\right) I: \bar{G} \rightarrow X$ and $A=I-\lambda_{0} U: \bar{G} \rightarrow X$, where $\lambda_{0} \in E_{\lambda}=$ $\{\lambda \in(0,1) \mid \lambda k<1\}$. Since $\lambda_{0} U: \bar{G} \rightarrow X$ is $k_{0}$-set-contractive with $k_{0}=\lambda_{0} k<1$ and $I-\lambda_{0} U$ satisfies the inequality (4.6), it follows that $A=I-\lambda_{0} U$ satisfies all the conditions of Theorem $1^{\prime}$. Indeed, the fact that $A$ is a continuous oneto-one map of $\bar{G}$ into $X$ such that $A(G)=D$ is bounded, $A(\bar{G})$ is closed, and $A^{-1}$ is a continuous map of $A(\bar{G})$ into $X$ follows from (4.6) while the fact that $A(G)$ is open follows from the invariance of domain theorem established in [29]. Furthermore, (4.6) implies that $\|F(x)-F(y)\| \leq\|A(x)-A(y)\|$ for $x, y \in \bar{G}$ and therefore the map $T=F A^{-1}: \bar{D} \rightarrow X$ is nonexpansive and, in particular, 1 -setcontractive. We shall now show that $T=F A^{-1}$ satisfies conditions (a') and (c') of Theorem 1'.

To see that $\left(\mathrm{a}^{\prime}\right)$ holds for some $y_{0}$ in $G$ note first that since $\lambda_{0} U$ satisfies conditions (a) and (c) of Theorem 1 for $x_{0}=0$, it follows that $\lambda_{0} U$ has a fixed point $\tilde{x}$ in $\bar{G}$ (i.e., $A(\tilde{x})=0$ ) with $\tilde{x}$ lying in $G$ because $\lambda_{0}$ lies in $(0,1)$ and $U$ satisfies (iii). Hence 0 lies in $D=A(G)$. We choose $\tilde{x}$ for $y_{0}$ in condition (a') of Theorem $1^{\prime}$ and observe that if $F(y)-A(\tilde{x})=\alpha(A(y)-A(\tilde{x}))$ (i.e., 
$F(y)=\alpha A(y))$, for some $y$ in $\partial G$, then $U(y)=\left(\left(\alpha+\lambda_{0}-1\right) / \alpha \lambda_{0}\right) y$ and, therefore, $\left(\alpha+\lambda_{0}-1\right) / a \lambda_{0} \leq 1$ by condition (iii). Since $\lambda_{0}$ lies in $(0,1)$, the latter inequality implies that $a \leq 1$, i.e., ( $\left.a^{\prime}\right)$ of Theorem $1^{\prime}$ holds. Now, condition (c') follows from (iv) and the fact that $F-A=\lambda_{0}(U-I)$.

Thus, by Theorem $1^{\prime}$, there exists $x$ in $\bar{G}$ such that $F(x)-A(x)=0$ or, equivalently, $x-U(x)=0$. Q.E.D.

Remark 4.4. Since every Lipschitzian map $U: \bar{G} \rightarrow X$ is $k$-set-contractive for $k$ equal to the Lipschitz constant and since the hypothesis that $(I-U)(\bar{D})$ is closed implies our condition (iv), Theorem 1 in [18] is a special case of our Theorem 4.1. We add in passing that the results in [18] were also deduced from the author's Theorem 7 in [34].

To obtain a result analogous to The orem 4.1 but without the assumption (iv) we first establish the following lemma.

Lemma 4.1. Let $X$ be a Banach space, $D$ a bounded open subset of $X$ with 0 in $D$, and $U$ a k-set-contractive map of $\bar{D}$ into $X$ for some $k \geq 0$ with

$$
(U(x), J(x)) \leq(x, J(x)) \text { for } x \text { in } \partial D .
$$

Then there exists an $r>0$ such that $(I-\lambda U)(\bar{D}) \supset \bar{B}(0,(1-\lambda) r)$ for any fixed $\lambda$ in $E_{\lambda}$.

Proof. First note that since $D$ is open and $0 \in D$ there exists a number $r>0$ such that $B(0, r) \subseteq D$. We shall show that Lemma 4.1 is valid for this particular $r$. Let $\lambda$ be any fixed number in $(0,1)$ such that $\lambda U$ is $k_{0}$-set-contractive with $k_{0}=\lambda k<1$, and let $f$ be any point in $\bar{B}(0,(1-\lambda) r)$. Note that the mapping $U_{f}=\lambda U+f: \bar{D} \rightarrow X$ is $k_{0}$-set-contractive and thus, in particular, $U_{f}$ is 1 -set-contractive and satisfies condition (c) of Theorem 1. Furthermore, $U_{f}$ satisfies condition (a) of Theorem 1 . Indeed, if $U_{f}(x)=\alpha x$ for some $x$ in $\partial D$, then condition (iii) and the definition of $r$ imply that for all $x$ in $\partial D$ we have

$$
\begin{aligned}
a(x, J(x)) & =\left(U_{f}(x), J(x)\right)=\lambda(U(x), J(x))+(f, J(x)) \leq \lambda(x, J(x))+\|f\|\|J(x)\| \\
& \leq \lambda(x, J(x))+(1-\lambda) r\|J(x)\| \leq \lambda(x, J(x))+(1-\lambda)\|x\|\|J(x)\|=(x, J(x)) .
\end{aligned}
$$

Thus, $\alpha \leq 1$ and so, by Theorem 1 for $x_{0}=0, U_{f}$ has a fixed point in $\bar{D}$, i.e., $(I-\lambda U)(\bar{D}) \supset \bar{B}(0,(1-\lambda) r)$. Q.E.D.

Now, if in Theorem 4.1 we strengthen the hypotheses on $X, G$, and/or $U$, then the condition (iv) in Theorem 4.1 can be omitted. To accomplish this we start with the following definitions.

Following [18] we say that $U: X \rightarrow X$ is strongly pseudo-contractive on $X$ relative to $G \subset X$ if for each $x$ in $X$ and $r>0$ there exists a number $a_{r}(x)<1$ such that 


$$
\|x-y\| \leq a_{r}(x)\|(1+r)(x-y)-r(U(x)-U(y))\|, \quad y \in G, x \in X .
$$

If in the above definition it is assumed that $U$ is defined only on $G$ and that (4.7) holds only for $x$ in $G$, then we shall say that $U: G \rightarrow X$ is strongly pseudo-contractive on $G$.

Theorem 4.2. Let $X$ be a Banach space and $U$ a k-set-contractive (for some $k \geq 0)$ map of $\bar{B}(0, q)$ into $X$ such that

$$
(U(x), J(x)) \leq(x, J(x)) \text { for } x \text { in } \partial B(0, q)
$$

Then $U$ bas a fixed point in $\bar{B}(0, q)$ provided any one of the following two conditions bold:

(A) $X$ is reflexive and $U: \bar{B} \rightarrow X$ is strongly pseudo-contractive on $\bar{B}$.

(B) $X$ is uniformly convex and $U: \bar{B} \rightarrow X$ is pseudo-contractive on $\bar{B}$.

Proof. It follows from Lemma 4.1 for $D=B(0, q)$ and for $r=q$ that $(I-\lambda U)(\bar{B}(0, q)) \supset \bar{B}(0,(1-\lambda) q) \equiv \bar{B}_{\lambda}$ for every $\lambda$ in $E_{\lambda}$. Now, let $\lambda_{0}$ be a fixed number in $E_{\lambda}$, let $r_{0}>0$ be such that $\lambda_{0}=r_{0} /\left(1+r_{0}\right)$, and for each $x$ in $\bar{B}(0, q)$ let $\alpha_{r_{0}}(x)=\alpha(x)$ be a number in $(0,1)$ such that

$$
\left(1-\lambda_{0}\right)\|x-y\| \leq \alpha(x)\left\|U_{\lambda_{0}}(x)-U_{\lambda_{0}}(y)\right\|, \quad y \in \bar{B}(0, q),
$$

with $\alpha(x)<1$ for each $x$ in $\bar{B}(0, q)$ when $U$ is strongly pseudo-contractive on $\bar{B}$, and with $\alpha(x) \equiv 1$ for all $x$ in $\bar{B}(0, q)$ when $U$ is pseudo-contractive on $\bar{B}$, where $U_{\lambda_{0}}=I-\lambda_{0} U$. It follows that $U_{\lambda_{0}}^{-1}$ exists, $U_{\lambda_{0}^{-1}}^{-1}\left(\bar{B}_{\lambda_{0}}\right) \subset \bar{B}(0, q)$ and the mapping $T_{\lambda}=(1-\lambda) U_{\lambda}^{-1}: \bar{B}_{\lambda_{0}} \rightarrow \bar{B}_{\lambda_{0}}$ is such that

$$
\left\|T_{\lambda_{0}}(x)-T_{\lambda_{0}}(y)\right\| \leq \alpha(x)\|x-y\| \text { for } x, y \text { in } \bar{B}_{\lambda_{0}} \text {. }
$$

Now, it follows from (4.8) that when $U$ is strongly pseudo-contractive on $\bar{B}(0, q)$, then $T_{\lambda_{0}}$ is a generalized contraction of $\bar{B}_{\lambda_{0}}$ into $\bar{B}_{\lambda_{0}}$ and therefore, since $X$ is reflexive, Theorem 2.5 implies the existence of a (unique) $x$ in $\bar{B}_{\lambda_{0}}$ such that $T_{\lambda_{0}}(x)=x$. On the other hand, when $U$ is pseudo-contractive on $\bar{B}$, then it follows from (4.8) that $T_{\lambda_{0}}$ is a nonexpansive map of $\bar{B}_{\lambda_{0}}$ into $\bar{B}_{\lambda_{0}}$ and therefore, since $X$ is uniformly convex, Theorem 2.2 implies the existence of an $x$ in $\bar{B}_{\lambda_{0}}$ such that $T_{\lambda_{0}}(x)=x_{0}$. In either case we see that $U_{\lambda_{0}^{-1}(x)=x /\left(1-\lambda_{0}\right)}$ from whence on setting $x^{1}=\left(1-\lambda_{0}\right)^{-1} x$ we find that $U\left(x^{1}\right)=x^{1}$ with $x^{1}$ in $\bar{B}(0, q)$. Q.E.D.

Remark 4.5. In case $U$ is Lipschitzian, Theorem 4.2 has first been proved by Browder and Petryshyn [8] for the case when $X$ is a Hilbert space and $U$ pseudo-contractive and then extended by Kirk [24] to the case when $X$ is 
uniformly convex and $U$ pseudo-contractive with $U(\partial B) \subset \bar{B}$. In its present form Theorem 2.4 has been proved in [36] for the case when $X$ and $U$ satisfy condition (B). Theorem 4.2 for $X$ and $U$ satisfying condition (A) appears to be new.

If in Theorem 4.2 we assume that $U$ is defined on all of $X$, then the following result holds, which for Lipschitzian mappings is due to Gatica and Kirk [18].

Theorem 4.3. Let $X$ be a Banach space, $D$ a bounded open convex subset of $X$ with 0 in $D$, and $U$ a mapping of $X$ into $X$ such that

$\left(a_{0}\right)$ If $U(x)=a x$ for some $x$ in $\partial D$, then $a \leq 1$.

Then $U$ bas a fixed point in $\bar{D}$ provided any one of the following two conditions bold:

$\left(A_{0}\right) X$ is reflexive and $U$ is Lipschitzian and strongly pseudo-contractive on $X$ relative to $\bar{D}$.

$\left(\mathrm{B}_{0}\right) X$ and $X^{*}$ are uniformly convex and $U$ is a map which is both k-set-contractive for some $k \geq 0$ and pseudo-contractive.

Proof. In view of Theorem 4.1 we need only show that in both cases $U$ satisfies condition (iv) of The orem 4.1, i.e., that if $\left\{x_{n}\right\}$ is a sequence in $\bar{D}$ such that $x_{n}-U\left(x_{n}\right) \rightarrow 0$ as $n \rightarrow \infty$, then there exists an $x$ in $\bar{D}$ such that $x-$ $U(x)=0$. Since $X$ is reflexive and $D$ is convex, the map $U$ will, of course, have the above property if in both cases we show that $I-U$ is demiclosed. The latter fact has been established by Browder [6] for the case when $X, X^{*}$, and $U$ satisfy condition $\left(B_{0}\right)$ and by Gatica and Kirk [18] for the case when $X$ and $U$ satisfy condition $\left(A_{0}\right)$. Q.E.D.

We complete this section with the following result which is related to Theorem $4.3\left(\mathrm{~B}_{0}\right)$ and whose proof is based on Theorem 8 in [36] and Corollary 2.1 .

Theorem 4.4. Let $X$ be a uniformly convex Banach space and let $U$ be a mapping of $X$ into $X$ which is both k-set-contractive for some $k .0$ and pseudocontractive. If there exists a bounded open convex subset $D$ of $X$ with 0 in $D$ such that

$\left(\mathrm{a}_{0}\right)$

$$
U(x) \neq \lambda x \text { for } x \text { in } \partial D \text { and } \lambda>1,
$$

then $U$ has a fixed point in $X$.

Proof. Let $E_{\lambda}=\{\lambda \in(0,1) \mid \lambda k<1\}$ and let $\lambda_{0}$ be any fixed number in $E_{\lambda}$. Then $\lambda_{0} U$ is a $k_{0}$-set-contraction of $X$ into $X$ with $k_{0}=\lambda_{0} k<1$ and since $U$ is also pseudo-contractive on $X$ it follows from (4.6) that for $U_{\lambda_{0}}=I-\lambda_{0} U$ we have

$$
\left\|U_{\lambda_{0}}(x)-U_{\lambda_{0}}(y)\right\| \geq\left(1-\lambda_{0}\right)\|x-y\| \text { for all } x, y \text { in } x .
$$

Hence, by the author's Theorem 8 in [36], $U_{\lambda_{0}}$ is a continuous one-to-one 
mapping of $X$ onto $X$ with the continuous inverse $U_{\lambda_{0}}^{-1}$. Furthermore, it follows from (4.9) that if we set $T_{\lambda_{0}}=\left(1-\lambda_{0}\right) U \boldsymbol{\lambda}_{0}^{1}$, then $T_{\lambda_{0}}$ is a nonexpansive mapping of $X$ into $X$. Since $X$ is uniformly convex and $D$ is a bounded open convex subset of $X$ with 0 in $D$, to prove that $T_{\lambda_{0}}$ has a fixed point in $\bar{D}$, by Corollary 2.1 it suffices to show that $T_{\lambda_{0}}$ satisfies condition (a) of Theorem 1 on $\partial D$. Now, since $U_{\lambda_{0}}$ is a one-to-one mapping of $\bar{D}$ into $X$ such that $U_{\lambda_{0}}(\bar{D})$ is open, $U_{\lambda_{0}}(\bar{D})=\overline{U_{\lambda_{0}}(D)}$, and $\partial U_{\lambda_{0}}(D)=U_{\lambda_{0}}(\partial D)$, it follows that if $T_{\lambda_{0}}(x)=a x$ for some $x$ in $\partial D$, then $U_{\lambda}^{-1}(x)=a x /(1-\lambda)$ with $a x /(1-\lambda)$ lying in $\partial D$. Consequently, $U(\alpha x /(1-\lambda))=((\alpha+\lambda-1) / \lambda(1-\lambda))=(\alpha+\lambda-1) \alpha x / \alpha \lambda(1-\lambda)$ from which, in view of $\left(\mathrm{a}_{0}\right)$, it follows that $\alpha \leq 1$. Hence, by Corollary 2.1 , there exists $x$ in $\bar{D}$ such that $T_{\lambda}(x)=x$ or $U\left(x^{\prime}\right)=x^{\prime}$ with $x^{\prime}=(1-\lambda)^{-1} x$. Q.E.D.

5. Application to maps satisfying suitable growth conditions. In this section Theorem 1 is applied to equations involving linear adjoint abelian mappings $A$ : $X \rightarrow X$ and nonlinear mappings $T: X \rightarrow X$ which satisfy certain growth conditions. For the study of such equations in case $X$ is a Hilbert space and $T$ satisfies a compactness or a $P$-compactness condition (see [27], [33], [43]). These equations are related to abstract Hammerstein equations ([33], [43]).

Let $X$ be a Banach space and let $J: X \rightarrow 2^{X^{*}}$ be a normalized duality mapping. Following Stampli [42] we say that a bounded linear mapping $A$ of $X$ into $X$ is adjoint abelian if $A^{*} J=J A$. Clearly, if $X$ is a Hilbert space, then the adjoint abelian operators are precisely the selfadjoint ones. It has been shown in [42] that adjoint abelian operators possess many properties of selfadjoint operators. Thus, for example, if $A: X \rightarrow X$ is adjoint abelian, then its spectrum $\sigma(A)$ is real, its spectral radius $\rho(A)=\|A\|$, and $\left\|A^{n}\right\|=\|A\|^{n}$ for $n=1,2,3, \ldots$ (see [42] for further properties).

The basic result of this section is the following fixed point theorem.

Theorem 5.1. Let $X$ be a Banach space, $A$ a bounded linear and adjoint abelian mapping of $X$ into $X$, and $T$ a nonlinear mapping of $X$ into $X$ such that, for all $y$ in $X$.

$$
(T(y), J(y)) \leq a_{1}\|y\|^{2}+a_{2}\|y\|^{2 \eta} \quad\left(a_{2}>0,0<\gamma_{1}<1, a_{1}\|B\|<1 \text { if } a_{1}>0\right),
$$

where $B=A^{2}$. If, for each $r>0$, the mapping $A T A: \bar{B}(0, r) \rightarrow X$ is either 1 -setcontractive or 1-ball-contractive and if ATA satisfies condition (c) on $\bar{B}(0, r)$, then $B T$ bas a fixed point in $X$.

Proof. Since $A: X \rightarrow X$ is adjoint abelian and (5.1) holds for all $y$ in $X$, it follows that for all $x$ in $X$

$$
(A T A(x), J(x))=\left(T A(x), A^{*} J(x)\right)=(T A(x), J A(x)) \leq a_{1}\|A x\|^{2}+a_{2}\|A x\|^{2 \eta} \text {. }
$$


This and the equality $\|A\|^{2}=\left\|A^{2}\right\|$ imply the inequality

$$
(A T A(x), J(x)) \leq a_{1}\|B\|\|x\|^{2}+a_{2}\|B\|^{\eta}\|x\|^{2 \eta} \text { for } x \text { in } X .
$$

Since $a_{1}\|B\|<1$ if $a_{1}>0$, and $1-\eta>0$, it is easy to see that there exists a ball $\bar{B}(0, r)$ such that

$$
a_{1}\|B\| r^{2}+a_{2}\|B\|^{\eta} r^{2 \eta}<r^{2} \text { for } x \text { in } \partial B(0, r) .
$$

Indeed, any fixed $r>0$ satisfying the inequality

$$
r>\left\{a_{2}\|B\|^{\eta} /\left(1-a_{1}\|B\|\right)\right\}^{1 /(2-2 \eta)}
$$

has this property. Hence for any fixed $r>0$ satisfying the inequality (5.2) we have the relation $(A T A(x), J(x)) \leq\|x\|^{2}$ for $x$ in $\partial B(0, r)$. This implies that the mapping $A T A: \bar{B}(0, r) \rightarrow X$ satisfies condition (a) of Theorem 1 for $x_{0}=0$. Since, by our assumption, $A T A: \bar{B}(0, r) \rightarrow X$ is either 1 -set-contractive or 1 -ballcontractive which satisfies condition (c) of Theorem 1 on $\bar{B}(0, r)$, it follows from Theorem 1 that there exists a point in $x$ in $\bar{B}(0, r)$ such that $x-A T A(x)$ $=0$, i.e., $z-B T(z)=0$, where $z=A(x)$. Q.E.D.

Remark 5.1. In case $X$ is a Hilbert space Petryshyn and Tucker [33] obtained an analogous theorem for $P$-compact operators satisfying (5.1).

As consequences we deduce from The orem 5.1 the following two useful results.

Theorem 5.2. Let $X$ be a Banach space, $A$ a bounded linear and adjoint abelian mapping of $X$ into $X$, and $S$ a mapping of $X$ into $X$ sucb that

$$
\|S(x)-S(y)\| \leq l\|x-y\| \quad \text { for } x, y \text { in } X \text { and some } l \geq 0 \text {. }
$$

(a) If $C$ is a compact mapping of $X$ into $X,\|B\| l<1$, and $T=S+C$ : $X \rightarrow X$ satisfies the inequality (5.1) of Theorem 5.1, then the mapping BT bas a fixed point in $X$.

(b) If $X$ is uniformly convex, $C: X \rightarrow X$ completely continuous, $\|B\| l \leq 1$, and $T=S+C$ satisfies the inequality (5.1), then $B T$ bas a fixed point in $X$.

Proof. In view of Theorem 5.1, to prove Theorem 5.2 it suffices to show that in both cases, for each $r>0$, the map $A T A: \bar{B}(0, r) \rightarrow X$ is either 1 -setcontractive or 1 -ball-contractive and condition (c) of Theorem 1 holds on $\bar{B}(0, r)$.

(a) Suppose first that $C: X \rightarrow X$ is compact and $\|B\| l<1$. Since $A T A=$ $A S A+A C A$ and $A$ is a bounded linear mapping such that $\|A\|^{2}=\left\|A^{2}\right\|=\|B\|$ with $\|B\| l<1$, it follows that $A C A$ is compact and $A S A$ is contractive with its Lipschitz constant $k=\|B\| l$. Hence $A T A$ is $k$-set-contractive with $k<1$. This implies that for each $r>0$ the mapping $A T A: \bar{B}(0, r) \rightarrow X$ is 1 -set-contractive 
and that $A T A$ satisfies condition (c) on $\bar{B}(0, r)$ for each $r>0$.

(b) Since $X$ is uniformly convex and $C$ is completely continuous, to prove assertion (b) it suffices to consider the case when $\|B\| l=1$ because the case when $\|B\| l<1$ follows from (a). Now, if $\|B\| l=1$, then $A T A=A S A+A C A$ : $\bar{B}(0, r) \rightarrow X$ is clearly 1 -set-contractive for each $r>0$ and therefore it suffices to show that $A T A$ satisfies condition (c) of Theorem 1 on $\bar{B}(0, r)$ for each $r>0$.

Let $r>0$ be any fixed number and let $\left\{x_{n}\right\}$ be any sequence in $\bar{B}(0, r)$ such that $x_{n}-A T A\left(x_{n}\right) \rightarrow 0$. Since $X$ is uniformly convex and $\left\{x_{n}\right\} \subset B(0, r)$ we may assume that $x_{n}-x_{0}$ in $\bar{B}(0, r)$. The fact that $A$ is a bounded linear mapping of $X$ into $X$ and that $C$ is completely continuous imply that $A C A\left(x_{n}\right)$ $\rightarrow A C A\left(x_{0}\right)$ as $n \rightarrow \infty$. Hence $x_{n}-A S A\left(x_{n}\right) \rightarrow A C A\left(x_{0}\right)$ as $n \rightarrow \infty$. Since $x_{n} \rightarrow x_{0}$ in $\bar{B}(0, r), X$ is uniformly convex, and $A T A: \bar{B}(0, r) \rightarrow X$ is nonexpansive, a result of Browder [6] implies that $x_{0}-A S A\left(x_{0}\right)=A C A\left(x_{0}\right)$ or $x_{0}-$ $A T A\left(x_{0}\right)=0$, i.e., condition (c) holds on $\bar{B}(0, r)$. Q.E.D.

Remark 5.2. In case $X$ is a Hilbert space, then the identity $I$ is the unique norma lized dua lity mapping of $X$ into $X\left(=X^{*}\right)$ and every adjoint abelian operator $A$ is a selfadjoint mapping of $X$ into $X$ with $B=A^{2}$ obviously selfadjoint and positive. For the latter case our Theorem 5.1 yields the results of Krasnoselsky [27] and Vainberg [43] for certain Hammerstein equations for the case when $A$ is compact.

Theorem 5.3. Let $X$ be a reflexive Banacb space, $A$ a bounded linear and adjoint abelian mapping of $X$ into $X$, and $S$ a mapping of $X$ into $X$ such that to each $x$ in $X$ and $r>0$ there corresponds a number $\alpha_{r}(x) \geq 0$ such that

$$
\|S(x)-S(y)\| \leq a_{r}(x)\|x-y\| \text { for } y \text { in } \bar{B}(0, r) .
$$

If $C$ is a compact mapping of $X$ into $X,\|B\| a_{r}(x)<1$ for each $x$ in $X$ and $r>0$, and $T=S+C: X \rightarrow X$ satisfies the inequality (5.1) of Theorem 5.1, then $B T$ has a fixed point in $X$.

Proof. In view of Theorem 5.1, it suffices to show that for each $r>0$ the mapping $A T A: \bar{B}(0, r) \rightarrow X$ is 1 -set-contractive and satisfies condition (c) on $\bar{B}(0, r)$. First, since $A C A$ is compact, $A T A$ will be 1 -set-contractive if $A S A$ : $\bar{B}(0, r) \rightarrow X$ is 1 -set-contractive for each $r>0$. Now, it follows from the linearity and boundedness of $A$ and from (5.4) that for each $x$ in $X$ and $r>0$

$$
\begin{aligned}
\|A S A(x)-A S A(y)\| & \leq\|A\|\|S A(x)-S A(y)\| \leq\|A\| a_{r}(A x)\|A x-A y\| \\
& \leq s_{r}(x)\|x-y\| \text { for } y \text { in } \bar{B}(0, r),
\end{aligned}
$$

where $s_{r}(x) \equiv\|B\| \alpha_{r}(A x)<1$ for each $x$ in $X$ and $r>0$. Hence $A S A: X \rightarrow X$ is uniformly strictly contractive on $\bar{B}(0, r)$ relative to $X$. Consequently, 
$A S A: \bar{B}(0, r) \rightarrow X$ is 1 -set-contractive and so is $A T A$. Now, to show that ATA: $\bar{B}(0, r) \rightarrow X$ satisfies condition (c) on $\bar{B}(0, r)$, let $\left\{x_{n}\right\}$ be a sequence in $\bar{B}(0, r)$ such that $x_{n} \cdot A T A\left(x_{n}\right) \rightarrow 0$ as $n \rightarrow \infty$. Since $A$ is bounded and linear, $C$ is compact, $\left\{x_{n}\right\} \subset B(0, r)$, and $X$ is reflexive we may assume $x_{n} \rightarrow x_{0}$ in $\bar{B}(0, r)$, $A C A\left(x_{n}\right) \rightarrow A(y)$ in $X$ for some $y$ in $X$, and $x_{n}-A S A\left(x_{n}\right) \rightarrow A(y)$ in $X$ as $n \rightarrow \infty$. This and the arguments of Kirk [23] imply that $x_{n} \rightarrow x_{0}$ as $n \rightarrow \infty$ so that $x_{0}-A S A\left(x_{0}\right)=A(y)$. But then $A C A\left(x_{n}\right) \rightarrow A C A\left(x_{0}\right)=A(y)$ and, therefore, $x_{0}-A T A\left(x_{0}\right)=0$, i.e., ATA satisfies condition (c) on $B(0, r)$. Q.E.D.

\section{BIBLIOGRAPHY}

1. M Altman, A fixed point theorem in Banach spaces, Bull. Acad. Polon. Sci. Cl. III 5 (1957), 19-22. MR 19, 297.

2. L. P. Belluce and W. A. Kirk, Fixed point theorems for certain classes of nonexpansive mappings, Proc. Amer. Math. Soc. 20 (1969), 141-146. MR 38 \#1663.

3. F. E. Browder, Nonexpansive nonlinear operators in a Banach space, Proc. Nat. Acad. Sci. U.S.A. 54 (1965/66). 1041-1044. MR 32 \# 4574.

4. — - Fixed point theorems for nonlinear semicontractive mappings in Banach spaces, Arch. Rational Mech. Anal. 21 (1966), 259-269. MR 34 \#641.

5. - Nonlinear mappings of nonexpansive and accretive type in Banach spaces, Bull. Amer. Math. Soc. 73 (1967), 875-882. MR 38 \#581.

6. - Semicontractive and semiaccretive nonlinear mappings in Banach spaces, Bull. Amer. Math. Soc. 74 (1968), 660-665. MR 37 \#5742.

7. - Nonlinear operators and nonlinear equations of evolution in Banach spaces, Proc. Sympos. Pure Math, Vol. 18, Part II, Amer. Math. Soc., Providence, R.I. (to appear).

8. F. E. Browder and W. V. Petryshyn, Construction of fixed points of nonlinear mappings in Hilbert space, J. Math. Anal. Appl. 20 (1967), 197-228. MR 36 \#747.

9. G. Darbo, Punti uniti in transformazioni a codominio non compatto, Rend. Sem. Mat. Univ. Padova 24 (1955), 84-92. MR 16, 1140.

10. J. Danes', Generalized concentrative mappings and their fixed points, Comment. Math. Univ. Carolinae 11 (1970), 115-136. MR 41 \# 7668.

11. D. E. Edmunds and J. R. L. Webb, Nonlinear operator equations in Hilbert spaces, J. Math. Anal. Appl. 34 (1971), 471-478. MR 43 \#6787.

12. D. E. Edmunds, Remarks on nonlinear functional equations, Math. Ann. 174 (1967), 233-239. MR 36 \#3180.

13. P. M. Fitzpatrick, A-proper mappings and their uniform limits, Ph. D. Thesis, Rutgers University, New Brunswick, N. J., 1971.

14. R. L. Frum-Ketkov, Mappings into a Banach sphere, Dokl. Akad. Nauk SSSR

175 (1967), 1229-1231 = Soviet Math. Dok1. 8 (1967), 1004-1006. MR 36 \#3181.

15. S. Fučík, Fixed point theorems for a sum of nonlinear mappings, Comment. Math. Univ. Carolinae 9 (1968), 133-143. MR 38 \#1567.

16. M. Furi and A. Vignoli, A fixed point theorem in complete metric spaces, Boll. Un. Mat. Ital. (4) 2 (1969), 505-509. MR 41 \#1034.

17. —, On a-nonexpansive mappings and fixed points, Atti Accad. Naz. Lincei Rend. Cl. Sci. Fis. Mat. Natur. (8) 48 (1970), 195-198. MR 43 \#5513.

18. J. A. Gatica and W. A. Kirk, Fixed point theorems for Lipschitzian pseudo-contractive mappings, Proc. Amer. Math. Soc. 36 (1972), 111-115.

19. D. Göhde, Zum Prinzip der kontraktiven Abbildung, Math. Nachr. 30 (1965), 251-258. MR $32 \# 8129$.

20. H. Hanani, E. Netanyaku and M. Reichaw-Reichbach, The sphere in the image, Israel J. Math. 1 (1963), 188-195. MR 29 \# 453.

21. V. Istrăteseu and A. Istrăteseu, On the theory of fixed points for some classes of mappings. IV (to appear). 
22. W. A. Kirk, A fixed point theorem for mappings which do not increase distances, Amer. Math. Monthly 72 (1965), 1004-1006. MR 32 \#6436.

23. - On nonlinear mappings of strongly semicontractive type, J. Math. Anal. Appl. 27 (1969), 409-412. MR 39 \#6128.

24. - Remarks on pseudo-contractive mappings, Proc. Amer. Math. Soc. 25 (1970), 820-823. MR 41 \#9074.

25. - Mappings of generalized contractive type (to appear).

26. M. A. Krasnosel'skii, Two remarks on the method of succesive approximations, Uspehi Mat. Nauk 10 (1955), no. 1 (63), 123-127. (Russian) MR 16, 833.

27. - Topological methods in the theory of nonlinear integral equations, GITTL, Moscow, 1956; English transl., Macmillan, New York, 1964. MR 20 \# 3464; MR 28 \# 2414.

28. K. Kuratowski, Topologie. Vol. 1, PWN, Warsaw, 1958; English transl., Academic Press, New York; PWN, Warsaw, 1966. MR 19, 873; MR 36 \# 840.

29. R. D. Nussbaum, The fixed point index and fixed point theorems for $k$-set-contractions, Ph. D. Thesis, University of Chicago, Chicago, Ill., 1969.

30. The radius of the essential spectrum, Duke Math. J. 37 (1970), 473-

478. MR 41 \# 9028.

31. - The fixed point index for local condensing maps, Ann. Mat.

Pura Appl. 89 (1971), 217-258.

32. W. V. Petryshyn, Fixed point theorems involving P-compact, semicontractive, and accretive operators not defined on all of Banach space, J. Math. Anal. Appl. 23 (1968), 336-354. MR 38 \# 588.

33. W. V. Petryshyn and T. S. Tucker, On the functional equations involving nonlinear generalized P-compact operators, Trans. Amer. Math. Soc. 135 (1969), 343-373. MR 40 \# 804.

34. W. V. Petryshyn, Structure of the fixed points sets of k-set-contractions, Arch. Rational Mech. Anal. 40 (1970/71), 312-328. MR 42 \#8358.

35. - Note on the structure of fixed point sets of 1-set-contractions, Proc. Amer. Math. Soc. 31 (1972), 189-194.

36. - Remarks on condensing and k-set-contractive mappings, J. Math. Anal. Appl. Appl. 39 (1972), 717-741.

37. J. Reinermann, Fixpunktsätze vom Krasnoselski-Typ, Math. Z. 119 (1971), 339-344. MR 43 \#3873.

38. E. Rothe, Zur Theorie der topologischen Ordnung und der Vektorfelder in Banachschen Raumen, Compositio Mat. 5 (1937), 177-197.

39. B. N. Sadovskil, On a fixed point principle, Funkcional. Anal. i Priložen. 1 (1967), no. 2, 74-76. (Russian) MR 35 \# 2184.

40. - Measures of noncompactness and condensing maps, Problemy Mat. Anal. Slož. Sistem 2 (1968), 89-119.

41. J. Schauder, Der Fixpunktsatz in Funktionalraumen, Studia Math. 2 (1930), $171-180$.

42. J. G. Stampfli, Adjoint abelian operators on Banach space, Canad. J. Math. 21 (1969), 505-512. MR 39 \#807.

43. M. M. Vaínberg, Variational methods for the study of non-linear operators, GITTL, Moscow, 1956; English transl., Holden-Day, San Francisco, Calif., 1964. MR 19, 567; MR 31 \#638.

44. G. M. Vaínikko and B. N. Sadovskií, On the degree of (ball) condensing vector fields, Problemy Mat. Anal. Slož. Sistem. 2 (1968), 84-88. (Russian)

45. J. R. L. Webb, Fixed point theorems for nonlinear semicontractive operators in Banach spaces, J. London Math. Soc. (2) 1 (1969), 683-688. MR 40 \#3392.

46. - Mapping and fixed ponit theorems for nonlinear operators in Banach spaces, Proc. London Math. Soc. (3) 20 (1970), 451-468. MR 42 \#17.

47. - Remarks on k-set-contractions, Boll. Un. Mat. Ital. 4 (1971), 614-629. 
48. S. Yamamuro, Some fixed point theorems in locally convex linear spaces, Yokohoma Math. J. 11 (1963), 5-12. MR 29 \#5095.

49. P. P. Zabrelko and M. A. Krasnosel'skil, A method for producing new fixed point theorems, Dokl. Akad. Nauk SSSR 176 (1967), 1233-1235 = Soviet Math. Dokl. 8 (1967), 1297-1299. MR 36 \#3183.

50. P. P. Zabreǐko, R. I. Kačurovskiǐ and M. A. Krasnosel'skiy̌, On a fixed point principle for operators in Hilbert space, Funkcional. Anal. i Priložen. 1 (1967), no. 2, 93-94. (Russian) MR 35 \#3505.

DEPARTMENT OF MATHEMATICS, RUTGERS UNIVERSITY, NEW BRUNSWICK, NEW JERSEY 08903 